\title{
Analysis of Automatic Control Function Effects on Vertical Tail Plane Critical Load Conditions
}

\author{
Thiemo M. Kier*, Reiko Müller ${ }^{\dagger}$, and Gertjan Looye ${ }^{\ddagger}$ \\ German Aerospace Center, DLR \\ Institute of System Dynamics and Control \\ 82234 Wessling, GERMANY \\ \{Thiemo.Kier, Reiko.Müller, Gertjan.Looye\}@dlr.de
}

\begin{abstract}
This paper presents a modelling scheme suitable for loads analysis of maneuvers and gusts of flexible aircraft with active control systems. In contrast to most ongoing research the component to be investigated is not the wing but the vertical tail plane (VTP). Critical load conditions for vertical tail plane include yawing maneuver conditions as well as discrete lateral gusts. A new rudder reversal load condition features a three full reversals of the rudder pedal input instead of just one step input and a mere return to neutral. This condition, where resulting loads are considered ultimate, was mainly motivated by wake vortex encounters during which the pilots made excessive use of the rudder. The design loads resulting from all conditions are heavily influenced by the flight control system, the underlying control law design method, and associated control law parameters. This gives rise to interesting trade-offs between handling qualities and loads sizing the VTP structure. Therefore, in this paper the influence of different types of lateral control laws on the loads of the different gust and maneuver conditions for certification as specified by the authorities is analysed. The control laws considered vary from basic yaw damping with rudder travel limitation to full roll and yaw command augmentation systems. From a design methodology point of view, classical and (incremental) Nonlinear Dynamic Inversion-based methods are analysed.
\end{abstract}

\section{Introduction}

The sizing loads for the aircraft structural components are determined by the so-called flight loads analysis. Objective of this analysis is to determine the dynamic loads that may occur during flight operations over the life time of an aircraft type. Flight loads analysis involves the simulation of many different load conditions at various points in the flight envelope, at different payload/fuel combinations and under normal conditions as well as under failure cases, which may amount to well over 1e5 load cases to be considered. Hence, the simulation models must be fast, yet sufficiently accurate to reliably simulate and assess all the different combinations that might become critical ("loop-capability"). The underlying computational analyses and the development of suitable models therefore constitute a specialized field of engineering within aircraft over-all design.

Loads predicted from the above analyses directly influence structural sizing and the eventual structural mass of the aircraft. Advanced construction methods, materials and the use of active control technologies allow for substantial reduction of flight loads and therefore reduction of structural weight. As the most substantial components in terms of structural weight, the research community is typically focused on prediction and reduction loads on the wings. Nevertheless, the vertical tail plane (VTP) is a highly relevant component and probably even more challenging from a flight loads point of view. As a key component for stability and control of the aircraft, the critical loads of the VTP are also heavily influenced by the flight control laws. In aircraft types with mechanically controlled rudder(s), these are especially the yaw damper function and the flight-condition dependent limitation of the rudder travel. Modern full fly-by-wire aircraft types provide side slip or lateral load factor command functions via the yaw axis. This naturally leads to a tradeoff between manoeuvre and gust loads, and achievable handling qualities.

The VTP is the component most susceptible to overload conditions. There are therefore many VTP-relevant load conditions specified in [1], such as the yawing manoeuvre (CS 25.351), the one engine out failure condition (CS 25.367), as well as discrete lateral gusts and continuous turbulence (CS 25.341). Past flight incidents [2] initiated a new

\footnotetext{
*Team leader Flight Dynamics and Loads, member AIAA

${ }^{\dagger}$ Research Engineer, member AIAA

${ }^{\ddagger}$ Head of Dept. Aircraft Systems Dynamics, member AIAA
} 
rule making process by the responsible authorities EASA [3] and FAA [4], where aircraft encountered wake vortex turbulence. The induced aircraft motion enticed the pilots to excessive use of the rudder, which in turn resulted in loads exceeding the design limit loads of the VTP [2]. The new paragraph CS 25.353 specifies a full-pedal command followed by three rudder reversals at the maximum sideslip angle before returning to neutral. This new paragraph is effective with the EASA CS-25 Amendment 22[1], released on the 5th November 2018. At the time of this writing the rule was in the final rule stage at the FAA

In our paper [5] we performed a detailed analysis of this new condition in comparison with the discrete gust and yawing manoeuvre ones based on a realistic model of a large passenger aircraft. We also compared the resulting VTP loads with simulated wake vortex encounters, all of them both for the open-loop case and in the presence of a yaw damper. In this paper we will focus on the influence of various types of fly-by-wire control functions and underlying design methodologies.

The main driver of this work has been the rapid development and growing number of application of nonlinear control methods such as Nonlinear Dynamic Inversion (NDI) and its growingly popular incremental variant (iNDI). DLR has been first to test iNDI on a CS-25 class passenger aircraft in co-operation with TU-Delft [6, 7] and simultaneously started analyzing flight loads implications on highly representative models of large passenger aircraft. From a design point of view, NDI uses nonlinear model equations to basically cancel nonlinear dynamic behaviour as part of control deflection commands. INDI is based on the same principle, yet uses angular acceleration commands to determine these terms. The inherent difference is that NDI leaves substantial degrees of freedom in effects to be directly compensated via model-based computation or outer loop control laws. Angular accelerations in INDI pick up and compensated for any disturbance and thus does not provide this freedom. This has a positive effect on tracking performance of commanded variables and may be exploited to reduce loads e.g. the wing roots [8], however, it may rise severe control activity and loads concerns at components like the VTP. This aspect will therefore be addressed in this paper as well.

This paper will first present a summary of an integrated modelling scheme for gust and manoeuver loads of a flexible controlled aircraft [9, 10], as well as analysis results presented in [5]. Next, an example function for side slip control based on NDI and INDI will be described, followed by implications of the functions in terms of loading of the VTP. As in [5], a subset of load conditions relevant for VTP structural sizing will be presented: the discrete lateral gusts (CS 25.341(a)), the yawing manoeuvre (CS 25.351) and the newly introduced rudder control reversal condition (CS 25.351). Based on these analyses, some preliminary, relevant conclusions on the effect of lateral directional control functions and the use of advanced control methods will be drawn.

\section{Integration of Loads Analysis Model}

The following section describes the general principles regarding the integration aspects of the loads analysis model, i.e., the structural model, the equations of motion, the external forces due to propulsion and the aerodynamics, and the flight control system. These equations are integrated in the loads environment VarLoads [11] and are expressed in closed form by the use of AIC matrices, i.e., no iteration between the structural and the aerodynamic model is necessary.

\section{A. Structural Dynamics, Equations of Motion and Load Recovery}

The starting point when setting up the equations of motion for a loads analysis model of a flexible aircraft is an Finite Element Model (FEM). This FEM usually consists of 100.000s of degrees of freedom (DoFs). Static condensation can be used to reduce the problem size by several orders of magnitude. The method employed is known as the Guyan reduction[12], where condensation points $(g-s e t)$ are placed along a loads reference axes. The mass distributions are prepared for the corresponding payload/fuel cases and connected to the $g-$ set points. Subsequently a modal analysis is carried out and only part of the modal basis is retained to further reduce the model size and computational cost.

The eigenvalues and eigenvectors define the generalized coordinates of the $h-s e t$. The zero eigenvalues represent the rigid body motion. The $h-s e t$ can be partitioned into six rigid body DoFs $(b-s e t)$ and a flexible part $(f-s e t)$. The rigid body mode shapes $\boldsymbol{\Phi}_{g b}$ and the retained modes of the eigenvector matrix $\boldsymbol{\Phi}_{g f}$ are used to generalized the equations of motion, which are given in the frequency domain by

$$
\left\{-\omega^{2}\left[\begin{array}{cc}
\mathbf{M}_{b b} & \mathbf{0} \\
\mathbf{0} & \mathbf{M}_{f f}
\end{array}\right]+\mathrm{j} \omega\left[\begin{array}{cc}
\mathbf{0} & \mathbf{0} \\
\mathbf{0} & \mathbf{B}_{f f}
\end{array}\right]+\left[\begin{array}{cc}
\mathbf{0} & \mathbf{0} \\
\mathbf{0} & \mathbf{K}_{f f}
\end{array}\right]\right\}\left[\begin{array}{l}
\mathbf{u}_{b} \\
\mathbf{u}_{f}
\end{array}\right]=\left[\begin{array}{c}
\boldsymbol{\Phi}_{g b}^{\mathrm{T}} \\
\mathbf{\Phi}_{g f}^{\mathrm{T}}
\end{array}\right] \mathbf{P}_{g}^{e x t}(\omega) .
$$

Note that the rigid body $b-$ set DoFs in Eq. (1) are defined in a earth fixed coordinate frame. 
A suitable set of equations of motion to account for large rigid body motions and linear flexibility is derived in the references [13, 14]. The nonlinear equations of motion describe the movement relative to a "mean axes" body reference frame. Equations of motion for an unrestrained flexible aircraft accounting for large rigid body motions are given by

$$
\begin{aligned}
{\left[\begin{array}{c}
\mathbf{m}_{b}\left(\dot{\mathbf{V}}_{b}+\boldsymbol{\Omega}_{b} \times \mathbf{V}_{b}-\mathbf{T}_{b E} \mathbf{g}_{E}\right) \\
\mathbf{J}_{b} \dot{\boldsymbol{\Omega}}_{b}+\boldsymbol{\Omega}_{b} \times\left(\mathbf{J}_{b} \boldsymbol{\Omega}_{b}\right) \\
\mathbf{M}_{f f} \ddot{\mathbf{u}}_{f}+\mathbf{B}_{f f} \dot{\mathbf{u}}_{f}+\mathbf{K}_{f f} \mathbf{u}_{f}
\end{array}\right]=\boldsymbol{\Phi}_{g b}^{\mathrm{T}} \mathbf{\Phi}_{g}^{\mathrm{ext}}(t) \mathbf{P}_{g}^{\mathrm{ext}}(t) }
\end{aligned}
$$

where $\boldsymbol{\Phi}_{g b}$ is the rigid body modal matrix about the center of gravity and in directions as customary in flight mechanics, i.e., x-forward, z-down. $\mathbf{V}_{b}$ and $\boldsymbol{\Omega}_{b}$ are the velocity and angular velocity vectors, respectively in the body frame of reference. The matrix $\mathbf{T}_{b E}$ transforms the gravitational vector from an earth fixed $(E)$ to the body fixed coordinate frame $(b)$ as a function of Euler angles.

In order to recover the nodal loads $\mathbf{P}_{g}$ for a subsequent sizing of the structure, the force summation method (FSM) [15] is employed. Thus, subtraction of the inertial loads $\mathbf{P}_{g}^{\text {iner }}$ from the external loads, yields

$$
\mathbf{P}_{g}=\mathbf{P}_{g}^{\text {ext }}-\underbrace{\mathbf{M}_{g g}\left\{\boldsymbol{\Phi}_{g b} \ddot{\mathbf{u}}_{b}+\boldsymbol{\Phi}_{g f} \ddot{\mathbf{u}}_{f}\right\}}_{\mathbf{P}_{g}^{\text {iner }}}
$$

In the case of the nonlinear equations of motion (2), the rigid body acceleration is given as

$$
\ddot{\mathbf{u}}_{b}=\left[\begin{array}{c}
\dot{\mathbf{V}}_{b}+\boldsymbol{\Omega} \times \mathbf{V}_{b}-\mathbf{T}_{b E} \mathbf{g}_{E} \\
\boldsymbol{\Omega}_{b}+\mathbf{J}_{b}^{-1}\left(\boldsymbol{\Omega}_{b} \times\left(\mathbf{J}_{b} \boldsymbol{\Omega}_{b}\right)\right)
\end{array}\right] .
$$

The FSM requires the external forces to be available in the structural DoF set $(g-s e t)$. This allows to account for the static part directly on the physical grid, and therefore has a good convergence behavior. Then cut loads can be computed by integrating the nodal loads along the loads reference axes of each aircraft component. The envelope of the cut loads is used as sorting criteria to obtain the critical load cases used for the structural sizing.

\section{B. Aerodynamic Model}

The major contribution to the external forces apart from the propulsion forces stem from the aerodynamics. So called Aerodynamic Influence Coefficient (AIC) matrices based on linear potential flow theory have classically been used for aeroelastic applications. The AIC matrices represent a linear relationship between the normalwash at the control point to the panel pressure, i.e., a change of the flow, normal to the panel surface at control point results in a change in pressure distribution. This allows to easily account for flexible deformation, which are simply treated as change in the normalwash vector $\mathbf{w}_{j}$.

The pressure coefficients are computed by

$$
\Delta \mathbf{c}_{\mathbf{p}_{j}}=\mathbf{Q}_{j j} \mathbf{w}_{j},
$$

where $\mathbf{Q}_{j j}$ is the so called AIC matrix. Traditionally, the Vortex Lattice and the Doublet Lattice Methods are used to obtain these AIC matrices. The Doublet Lattice Method provides the complex valued AIC matrix as function of reduced frequency $k=\frac{c_{r e f} / 2}{U_{\infty}} \omega$, which describe the unsteady aerodynamic transfer functions. In frequency domain calculations the complex AICs can be used directly. For time domain simulations, a Rational Function Approximation (RFA) [9, 16] is required to transform the AICs to the Laplace domain. The rational functions can then be cast in the form of a system of linear ordinary differential equations amenable to time integration.

The load transformation to panel reference point is done by integrating the pressures, which is mostly a simple multiplication with the aerodynamic box area. In some classical aerodynamic panel methods, additional moments occur due to an offset between control point and pressure application point, cf. [17]. These are accounted for by introducing rotational degrees of freedom in the aerodynamic panel $(k-s e t)$ and the respective moment arms into the integration matrix $\mathbf{S}_{k j}$. Multiplication with the dynamic pressure yields the aerodynamic forces.

$$
\mathbf{P}_{k}^{\text {aero }}=q_{\infty} \mathbf{S}_{k j} \mathbf{c}_{\mathbf{p}_{j}}
$$


Next, the boundary condition for the normalwash has to be considered:

$$
\mathbf{w}_{j}(k)=\left(\mathbf{D}^{\mathbf{x}}{ }_{j k}+\frac{\mathrm{d}}{\mathrm{d} t}\left(\frac{c_{r e f} / 2}{U_{\infty}}\right) \cdot \mathbf{D}_{j k}^{\mathbf{t}}\right) \mathbf{u}_{k}(t),
$$

where the matrix $\mathbf{D}^{\mathbf{x}}{ }_{j k}$ accounts for a change in downwash due to tilting of the normal vector with respect to the free stream direction and the matrix $\mathbf{D}_{j k}^{\mathbf{t}}$ for additional downwash due to movement of the boundary in direction of the panel normal. The factor $\frac{c_{r e f} / 2}{U_{\infty}}$ in equation (7) is needed due to the conversion from reduced to natural frequency. The vector $\mathbf{u}_{k}(t)$ represents the motion of the aerodynamic reference points.

When the nonlinear equations of motion are used, special attention to the boundary condition is required. The vectors $\mathbf{V}_{b}$ and $\boldsymbol{\Omega}_{b}$ are defined in a body fixed frame of reference. Hence, the steady deflection of rigid body modes does not induce aerodynamic loads. Therefore, the differentiation matrix $\mathbf{D}^{\mathbf{x}}$ needs to be canceled for the rigid body modes.

Finally, the aerodynamic loads have to be mapped to the structural degrees of freedom. The matrix connecting the displacements of the structural grid $(g-s e t)$ to the aerodynamic grid $(k-s e t)$ is called spline matrix $\mathbf{T}_{k g}$.

$$
\mathbf{u}_{k}=\mathbf{T}_{k g} \mathbf{u}_{g}
$$

This mapping is achieved by, e.g., employing radial basis functions such as the commonly used Infinite Plate Spline (IPS) [18]. The aerodynamic loads can be mapped back onto the structure with the transpose of the spline matrix, based on the principal of virtual work.

$$
\mathbf{P}_{g}^{\text {aero }}=\mathbf{T}_{k g}^{\mathrm{T}} \mathbf{P}_{k}^{\text {aero }}
$$

Similarly, the modal matrix $\mathbf{\Phi}_{g f}$ and its transpose connect the flexible part of the equations of motion (1) and (2) to the aerodynamic model.

\section{Rational Function Approximation}

The Doublet Lattice Method [19] provides aerodynamic matrices as tabulated values at discrete reduced frequencies. One possibility to make them amenable for time domain integration is the so called rational function approximation (RFA), where the frequency domain transfer functions are fit with suitable "rational" terms. These can then be Laplace transformed and cast in state space form. Many flavors of this method have been published in literature [16, 20, 21]. Most of these publications concentrate on approximation of the generalized aerodynamic matrices $\mathbf{Q}_{h h}$, i.e. the AIC matrices are already post-multiplied with the differentiation matrices (7) and the modal basis. This approach reduces the computational cost due to a smaller problem size.

In [9] an RFA fit of the AICs $\mathbf{Q}_{j j}(k)$ without prior multiplication with differentiation matrices was proposed, the so called "physical" RFA:

$$
\mathbf{Q}_{j j}(\hat{s})=\mathbf{Q}_{j j}^{\mathbf{0}}+\mathbf{Q}_{j j}^{\mathbf{1}} \hat{s}+\sum_{i=1}^{n_{p}} \mathbf{Q}_{j j}^{\mathbf{L}_{i}} \frac{\hat{s} \mathbf{I}}{\hat{s}+p_{i}},
$$

where $\hat{s}=s\left(\frac{c_{r e f} / 2}{U_{\infty}}\right)$ is the Laplace domain equivalent to the reduced frequency $k$. The reason for the presence of a second derivative in the classical RFA compared to the present formulation, is the additional time derivative in the downwash equation (7). The present, "physical" RFA (10) has several advantages over the approximation of the generalized aerodynamic forces, e.g., the fit is not tied to a particular mass case. But more importantly, the individual terms of the fit allow a physical interpretation: The term $\mathbf{Q}_{j j}^{\mathbf{0}}{ }_{j j}$ represents the quasi-steady term, $\mathbf{Q}^{\mathbf{1}}{ }_{j j}$ is the added mass (in incompressible flow), and the terms $\mathbf{Q}^{\mathbf{L}_{i}}{ }_{j j}$ with the predefined poles $p_{i}$, are responsible for the lagging behavior of the unsteady flow.

Since the input to the physical RFA is defined on the control point level, the gust velocity and the time lags associated with the penetration speed can be applied directly. The problematic approximation of the gust column can be omitted completely. Further, this fit also allows the consideration of a nonlinear position dependence of the wind field as demonstrated in [10], since the normalwash $\mathbf{w}_{j}$ can be computed online and fed into a realization of the ordinary differential equations (ODE) (11) of the unsteady aerodynamics in order to determine the so called lag states $\mathbf{x}_{L}$.

$$
\mathbf{x}_{L}=\frac{U_{\infty}}{c_{r e f} / 2} \mathbf{R} \mathbf{x}_{L}+\mathbf{E} \mathbf{w}_{j}
$$


The matrices $\mathbf{R}$ and $\mathbf{E}$ are stacked diagonal matrices, containing the poles $p_{i}$, respectively identity matrices. The splined aerodynamic forces including steady the unsteady parts are then

$$
\mathbf{P}_{g}^{\text {aero }}=\underbrace{\left(\mathbf{Q}_{g j}^{\mathbf{0}} \mathbf{w}_{j}\right)}_{\text {steady } \mathbf{P}_{g}^{s}\left(\mathbf{w}_{j}\right)}+\underbrace{\left(\mathbf{Q}_{g j}^{\mathbf{1}}\left(\frac{c_{\text {ref }} / 2}{U_{\infty}}\right) \mathbf{w}_{j}+\mathbf{D} \mathbf{x}_{L}\left(\mathbf{w}_{j}\right)\right)}_{\text {unsteady } \mathbf{P}_{g}^{u}\left(\mathbf{w}_{j}\right)},
$$

where $\mathbf{D}$ contains the coefficients $\mathbf{Q}^{\mathbf{L}_{i}}{ }_{g j}$ from the least squares procedure according to Roger [16]. With the "physical" RFA it is possible to discern between the steady and unsteady contribution of the aerodynamics, which is impossible when the fit is applied to the generalized aerodynamic forces.

\section{Flight Control System}

To realistically represent the behavior of an aircraft subjected to pilot inputs or atmospheric disturbances, the flight control system (FCS) also needs to be considered. The classical aeroelastic triangle [22] becomes an aeroservoelastic tetrahedron. The flight control system consists of sensors, which signals can then be processed by the flight control law implementations, and command control surface deflections via actuation systems. The flight control laws can alter the dynamics of the airframe and are present on all modern aircraft.

\section{Flight Control Laws}

The present flight control laws are designed according to the classical cascaded flight controller layout, where an inner loop captures fast states like aircraft orientation, and an outer loop or autopilot controlling slower states like flight path angle, course and speed. Since these variables change on different time scales, the two loops can be adjusted / tuned sequentially (instant attainment of faster states as seen by the outer loop and constant slower states as viewed from the inner loop). Furthermore, as longitudinal and lateral dynamics are only weakly coupled for a standard configuration fixed-wing aircraft, an additional separation into respective channels is possible in both loops.

For the considered scenarios, inner loop controllers for each the rolling, pitching and yawing motion with fixed structure have been implemented, while the outer loop / autopilot consists of the Total Energy Control System (TECS) and Total Heading Control System (THCS) formulations. The latter allow combined tracking of altitude and speed as well as course and sideslip angle respectively. In the case of the longitudinal autopilot/TECS, the energy conservation principle serves as basis for calculating pitch and throttle commands which balance potential and kinetic energy and regulate the total energy of the system.

The autopilot for the lateral motion / THCS similary adjusts roll angle and yaw rate commands in order to track commanded heading and sideslip angles, where the roll rate works to cancel the sum and the yaw rate to adjust the difference of the angle errors. Details on the concepts of TECS and THCS given in the original patent of Lambregts [23] and later adaptations [24, 25].

\section{Yaw Damper and Rudder Travel Limiter}

For the control of the yaw axis, the rudder control surface on the vertical tail plane is used. The rudder control surface has a variety of functions, where certain handling quality objectives must be met, e.g. to counteract crosswind or an engine failure during takeoff $\left(V_{\mathrm{MCg}}\right)$ on ground. To perform a crosswind approach and decrab the aircraft during the last phase of the landing to ensure alignment with the runway when flaring. When one engine is inoperative during fligh, the resulting yawing moment must be counteracted. This requirement is associated with the minimum control speeds $V_{\mathrm{MCa}}$ in the air and $V_{\mathrm{MCl}}$ during approach in landing configuration, where in clean and high lift configuration a sufficient yawing moment must be provided with no more than 5 degrees bank angle (CS 25.149). During manoeuvres, rudder deflection is required for turn coordination to avoid lateral load factors due to sideslip. Furthermore, the lateral flight control law increases the damping of unwanted flight characteristics such as the dutch roll mode. The dutch roll is an eigenmode of the flight mechanics where rolling and yawing motion are coupled in an unfavorable way. Sometimes additional lateral flight control functions are implemented to enhance passenger comfort, e.g. mitigating the excitation of structural modes such as lateral fuselage bending through rudder usage.

These lateral flight control functions are implemented in the so called yaw damper, which augments the pilot yawing command (pedal input).

Figure 1 depicts the block diagram of an implementation of such a function. The measured yaw rate $r_{m}$ is subtracted from a commanded yaw rate $r_{c}$ issued either by the pilot or autopilot. The resulting error is proportionally applied to 


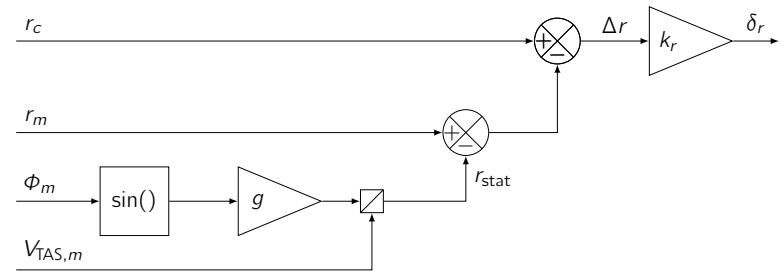

Fig. 1 Yaw damper, which subtracts the stationary yaw rate for turning flight $\left(r_{\text {stat }}\right)$ from measured yaw rate $r_{m}$. Similar to a high-pass filter, this yields the relevant frequencies of the Dutch-Roll motion.

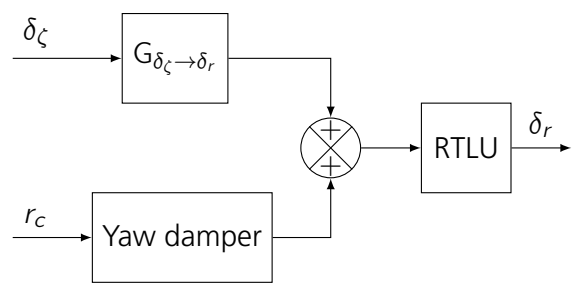

Fig. 2 Rudder actuation through commands from pedals and yaw damper. The function $\mathrm{G}_{\delta_{\zeta} \rightarrow \delta_{r}}$ translates pedal to rudder deflection commands

the rudder actuator as commanded yawing acceleration $k_{r} \cdot \Delta r$. However, since only disturbances due to the previously mentioned causes shall be damped, the stationary part of the yaw rate (which is for example present during turning flight) needs to be removed from the measured signal $r_{m}$. This can be done for example by employing a high-pass filter, or by directly subtracting the stationary turning rate $r_{\text {stat }}=\frac{g \cdot \sin \Phi_{m}}{V_{\text {TAS }}}$. The gain of the yaw damping function $k_{r}$ determines the degree of attenuation.

Large rudder deflections incur high loads on the VTP structure, therefore the deflection is limited by a so called Rudder Travel Limitation Unit (RTLU). The deflection limit is scheduled as a function of the calibrated flight speed $V_{\text {CAS }}$ to meet the pertaining handling quality requirements but at the same time avoid excessive loads on the VTP. It is applied to the sum of commands from pilot $\delta_{\zeta}$ and yaw damper $r_{c}$, as shown in Figure 2

\section{Critical Design Load Conditions for the Vertical Tail Plane}

Many paragraphs in the certification specifications are relevant for the structural sizing of the vertical tail, such as the one engine out condition (CS 25.367), lateral continuous turbulence (CS 25.341 (b)) as well as failure cases such

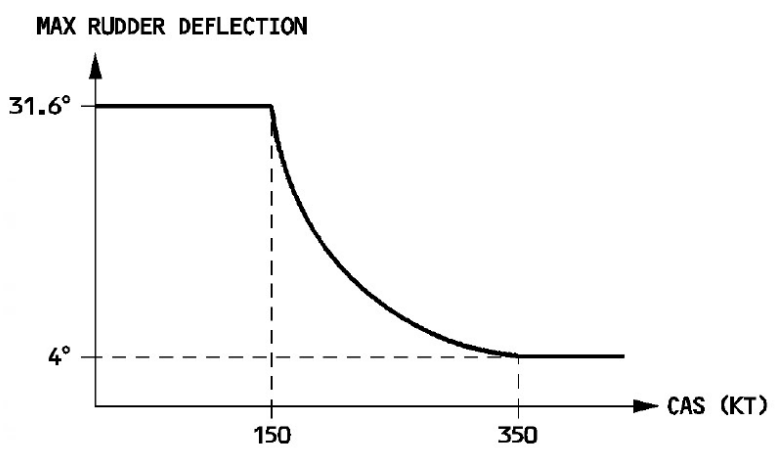

Fig. 3 Maximum rudder deflection in dependence of $V_{\mathrm{CAS}}$, used inside the RTLU 
as rudder runaways, oscillatory failures or force fighting of redundant rudder actuation systems. However, this paper focuses on the discrete tuned lateral gust (CS 25.341(a)) and the yawing manoeuvre condition (CS 25.351). These two conditions are exemplary for induced loads by external atmospheric disturbance, respectively by pilot control inputs.

Furthermore, the newly introduced rudder control reversal condition (CS 25.353) is described and contrasted to the existing yawing manoeuvre paragraph CS 25.351.

\section{A. Discrete Tuned Lateral Gust Condition}

The paragraph for the analysis of lateral gusts evolved from using a Pratt type formula to the rational analysis of a discrete gust with varying gust wave lengths. Thereby, the lateral gust regulations followed a similar path of evolution to the vertical gust paragraphs. The vertical gust were originally described by the so called Pratt gust [26], taking into account a sudden change of angle of attack due to the gust velocity, resulting in an additional load factor $n_{z}$ increment. The fact that the sharp edged gust induces more loads than a smooth gust profile was accounted for by an alleviation factor.

Later in JAR-25 change 13 from 5th October 1989 [27], a 1-cosine gust shape of a fixed gust length of 25 semi-chords was specified. Note that the mean geometric and not the mean aerodynamic chord is used as reference length. In JAR-25 change 14 from 27th May 1994 [28] the gust and turbulence paragraphs were harmonized [29], originally issued as so called orange paper amendments. The gust paragraph CS 25.341 was no longer solely responsible for symmetric vertical gust but also included the lateral gust conditions. The gust gradient lengths of 1-cosine shape now have to be varied between 30 and $350 \mathrm{ft}$. Furthermore section b) was introduced, a Continuous Turbulence Design Criteria where the response to the statistical von Karman power spectrum given in the frequency domain needs to be considered.

The lateral gust criterion in JAR-25 change 13 was originally formulated in paragraph JAR 15.351(b) Lateral Gusts. The Pratt type formula simply considered the side force $L_{t}$ due to a gust, where $K_{\mathrm{gt}}$ is the gust alleviation factor with the lateral mass ratio $\mu_{\mathrm{gt}}$.

$$
L_{\mathrm{t}}=\frac{K_{\mathrm{gt}} U_{\mathrm{de}} V S_{\mathrm{t}}}{498} \quad ; \quad K_{\mathrm{gt}}=\frac{0.88 \mu_{\mathrm{gt}}}{5.3+\mu_{\mathrm{gt}}} \quad ; \quad \mu_{\mathrm{gt}}=\frac{2 W}{\rho \overline{\mathrm{c}_{\mathrm{t}} g a_{\mathrm{t}} S_{\mathrm{t}}}}\left(\frac{K}{l_{\mathrm{t}}}\right)^{2}
$$

$U_{\mathrm{de}}$ is the derived gust velocity ( $\left.\mathrm{ft} / \mathrm{s}\right), \rho$ is the air density (slugs $\left./ \mathrm{ft}^{3}\right), W$ the aeroplane weight $(\mathrm{lb}), S_{\mathrm{t}}$ is the area of vertical tail $\left(\mathrm{ft}^{2}\right), \bar{c}_{\mathrm{t}}$ is the mean geometric chord of vertical surface ( $\mathrm{ft}$ ), $a_{\mathrm{t}}$ is the lift curve slope of vertical tail (per radian), $K$ the radius of gyration in yaw (ft), $l_{\mathrm{t}}$ is the distance from aeroplane c.g. to lift centre of vertical surface (ft), $g$ is the acceleration due to gravity ( $\left.\mathrm{ft} / \mathrm{s}^{2}\right)$ and $V$ is the aeroplane equivalent speed (knots).

Paragraph CS 25.341(a) nowadays features a discrete 1-cosine shaped gust profile. Gust gradient distances $H$ in the range $30 \mathrm{ft}$ to $350 \mathrm{ft}$ must be considered to determine the critical response, cf. Figure 4

$$
U=\frac{U_{\mathrm{ds}}}{2}\left(1-\cos \left(\frac{\pi S}{H}\right)\right)
$$

where the design gust velocity is given by

$$
U_{\mathrm{ds}}=U_{\mathrm{ref}} F_{\mathrm{g}}\left(\frac{H}{107}\right)^{\frac{1}{6}} .
$$

The reference gust velocity $U_{\text {ref }}$ is linearly interpolated as function of altitude, starting from $56 \mathrm{ft} / \mathrm{s}$ at sea level, $44 \mathrm{ft} / \mathrm{s}$ at $15000 \mathrm{ft}$ and $20.86 \mathrm{ft} / \mathrm{s}$ at $60000 \mathrm{ft}$. For the aeroplane design speed $V_{D}$ the reference gust velocity is half of this value.

Hence, the design gust velocity varies with the gradient distance and a so called flight profile alleviation factor, which depends on the design weights of the aircraft type. The value for the flight profile alleviation factor is

$$
F_{g}=\frac{1}{2}\left(1-\frac{z_{\mathrm{mo}}}{76200}+\sqrt{\frac{m_{\mathrm{MLW}}}{m_{\mathrm{MTOW}}} \tan \frac{\pi}{4} \frac{m_{\mathrm{MZFW}}}{m_{\mathrm{MTOW}}}}\right),
$$

where $z_{\mathrm{mo}}$ is the maximum operating altitude. All velocities are given in equivalent airspeed (EAS) and have to be converted to true airspeed (TAS) in the corresponding altitude.

For the gust load computations unsteady aerodynamics need to be taken into account. Gust loads analysis is usually carried out in the frequency domain, since the doublet lattice method readily provides the AIC matrices complex form as function of the reduced frequency parameter $k$. In this paper the analysis is done in the time domain, as it conveniently allows provision for nonlinearities in control systems. 


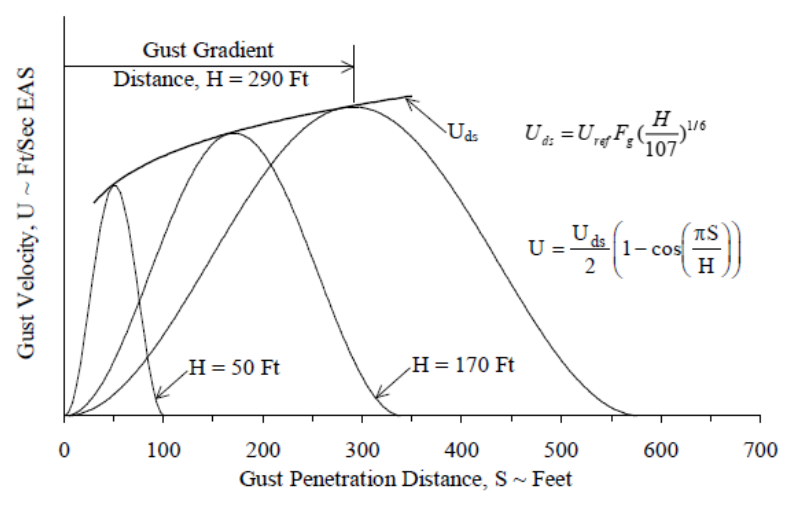

Fig. 4 gust shape from AMC 25.341 of CS-25 [1]

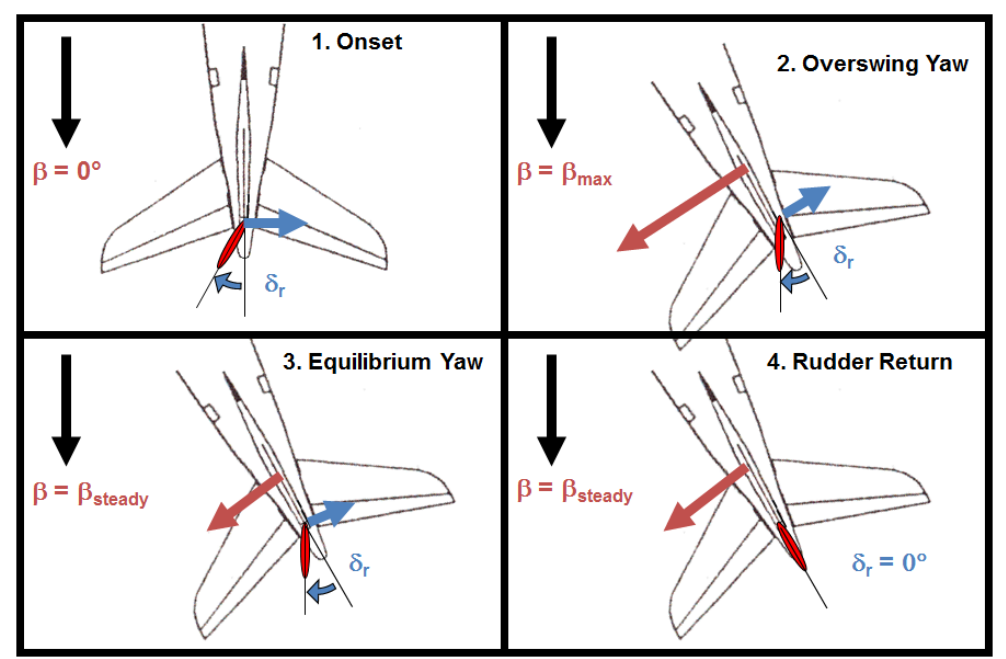

Fig. 5 Yawing manoeuvre: four phases

\section{B. Yawing Manoeuvre Condition}

The yawing manoeuvre is a pilot induced load condition. Besides the unsymmetrical loads due to engine failure of CS 25.367, which is not considered in this paper, the yawing manoeuvre results in the large torsional loads on the vertical tail plane structure due to pedal input of the pilot.

The yawing manoeuvre needs to be considered between the minimum control speed $V_{\mathrm{MC}}$ and the design dive speed $V_{\mathrm{D}}$. It can be characterized by four different phases:

1) Onset: Starting from level flight, the rudder is deflected by a sudden pilot pedal command.

2) Overswing: As a result of the rudder command the aircraft starts to yaw and a dynamic overswing resulting in a maximum sideslip angle occurs.

3) Equilibrium Yaw: Continuing the full rudder command, a state of constant sideslip is reached.

4) Rudder Return: Form the steady sideslip condition, the rudder command is returned to zero.

The occurring loads of the yawing manoeuvre heavily depend on the active lateral control law in particular the yaw damping function and the travel limit of the rudder control surface deflection.

\section{Rudder Control Reversal Condition}

A brand new paragraph is the CS 25.353 rudder control reversal condition. It is included in amendment 22 of the EASA CS 25, released on 5th November 2018. The FAA rule making process is the final stage, however an update of the 14 CFR Part 25 including the new paragraph has not been released yet (as of 20th May 2019). 


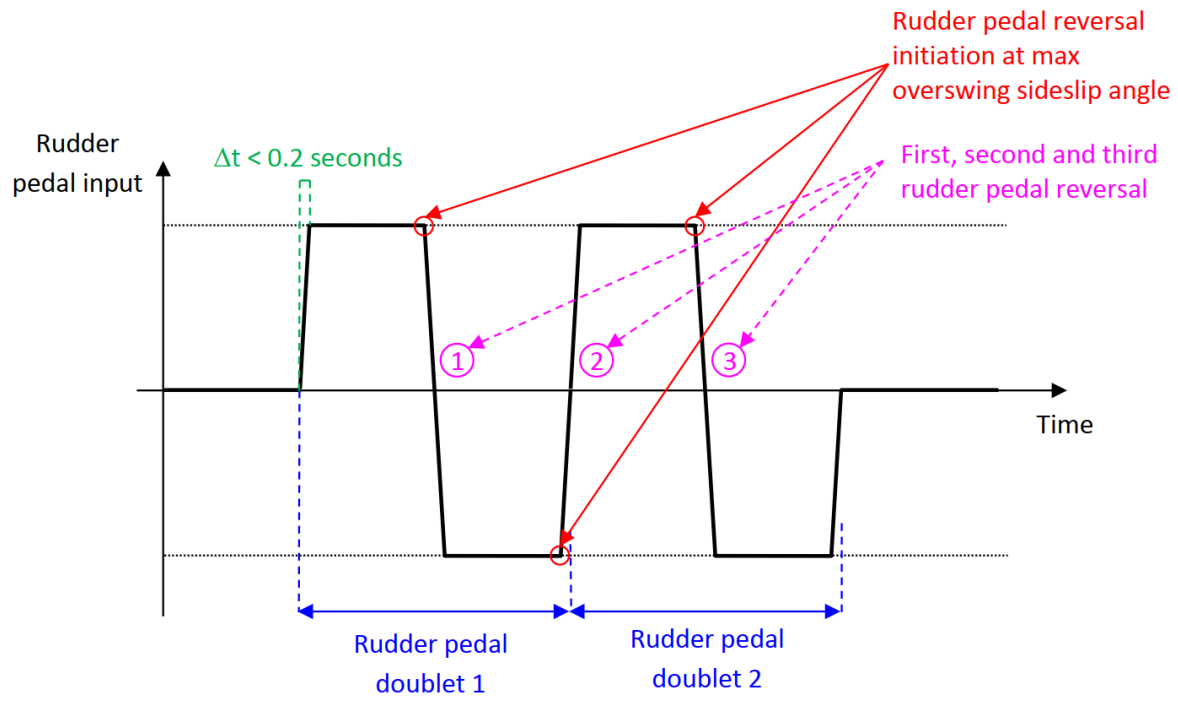

Fig. 6 : CS 25.353 Rudder control reversal load condition as depicted in [1]

The rudder reversal is a second yawing manoeuvre condition also involving rudder pedal inputs by the pilot. The chosen name seems to be somewhat unfortunate, since a reversal condition is usually associated with the aeroelastic phenomenon and not with an alternating pilot input, which might lead to a confusion. The new load condition consist of two full rudder doublets as opposed to only one rudder input as in CS 25.351. Furthermore, instead of reversing the controls at a steady sideslip angle, the rudder command has to be reversed at the maximum sideslip angle, which further increases the loads on the VTP. The onset of the manoeuvre of CS25.353 is addressed in subparagraph (a), the three control reversals in (b), (c) and (d), and finally the return of the rudder control to neutral in subparagraph (e), as depicted in Figure 6

In contrast to the yawing manoeuvre CS 25.351, only speeds between minimum control speed $V_{\mathrm{MC}}$ and design cruise speed $V_{\mathrm{C}}$ need to be considered.

The new load condition was introduced because incident investigations showed that pilots may make inadvertent or inappropriate rudder inputs during severe external disturbances, e.g. due to wake vortex encounters, in order to avoid upset flight conditions. Since the rate of occurrence for such incidences was deemed to be low, the loads resulting from such rudder reversals can be considered ultimate, i.e. the factor of safety of 1.5, as specified in CS 25.303, does not need to be applied.

\section{Simulation Results}

The model used for the present study is one of a generic long range aircraft with two engines. Only one mass case is considered and also only the flight at the $V_{C} / M_{C}$ intersection is covered. At this point in the flight envelope the gust velocity is not reduced and the dynamic pressure is large. Also the new rudder reversal manoeuvre condition only needs to be considered up to $V_{C}$. So the computed loads do not necessarily represent a design loads envelope but should be representative in terms of the resulting loads levels.

Nine different gust gradient lengths are considered for the discrete gust condition, where only a subset will be displayed individually for clarity. The yawing manoeuvre condition complements the gust loads for the design cases according to the status of CS-25 Amndt. 21 [30]. The resulting loads are then compared to those of the newly introduced the rudder control reversal condition.

As stated before the resulting loads are very sensitive to the chosen lateral control law. Therefore, different gains for the yaw damping function are assessed. One for a critically damped yawing motion $\left(k_{r}=2.0\right)$, i.e. no overswing in sideslip angle, one undamped control law without rudder action due to yaw rate $\left(k_{r}=0.0\right)$, and an intermediate damping gain with some overswing behavior $\left(k_{r}=0.5\right)$. Some qualitative remarks about the influence of the rudder travel limiter inhibiting the maximum deflection are made. 

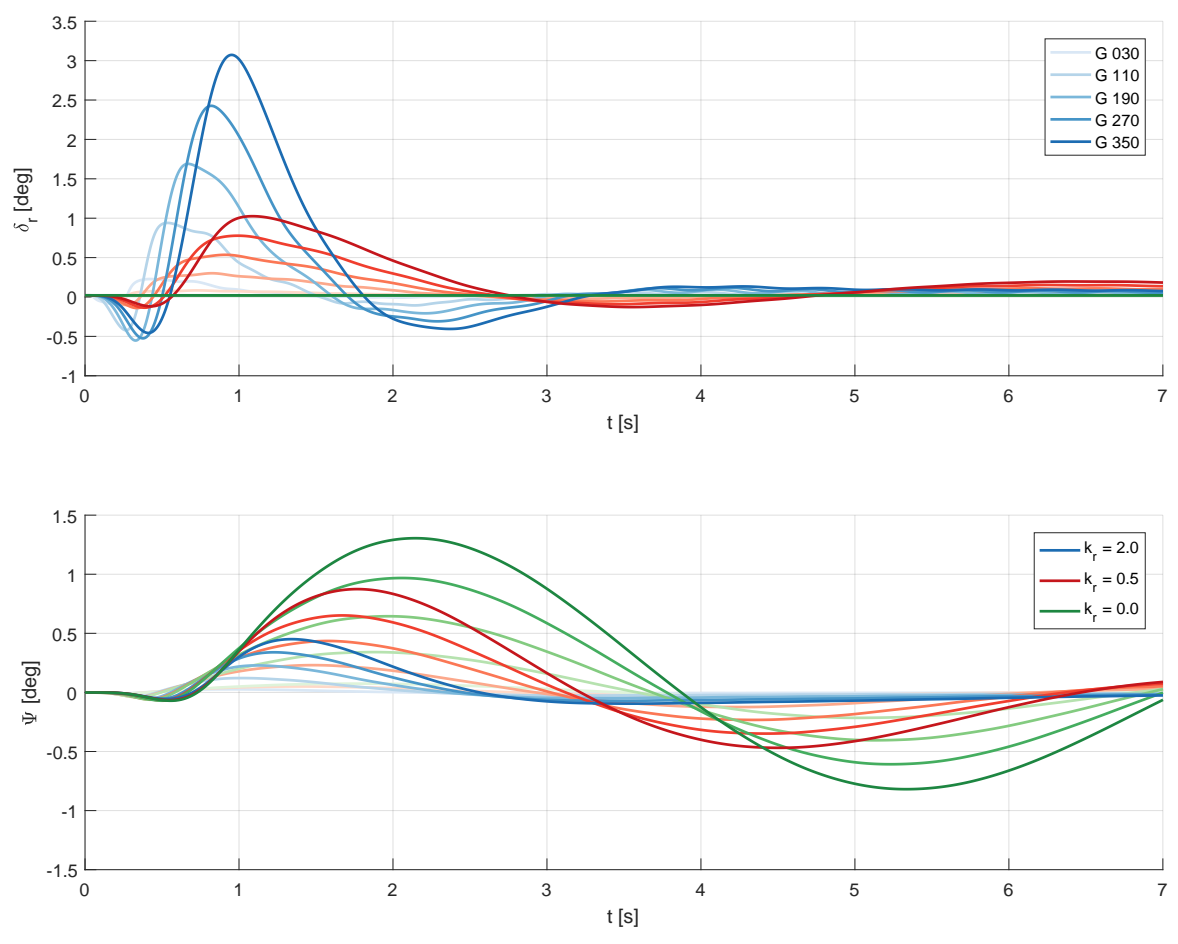

Fig. 7 Rudder deflection and heading angle during lateral discrete gust

The comparison for the three different yaw damper gains is based on the correlation of bending and torsion moment loads at the VTP root. Furthermore, a hierarchy of the critical cases for the shear, bending and torsion moment along the VTP component axis is shown.

\section{A. Lateral Discrete Gust}

First, the lateral gust hits the front of the aircraft resulting in a yawing motion away from the gust. When the gust arrives at the vertical tail, the aircraft nose points into the direction of the gust. The yaw damper deflects the rudder accordingly, as shown in Figure 7. Longer gradient distances are accompanied by larger gust velocities. Therefore, larger yaw rates are induced which in turn result in more rudder deflection. Another contributing factor is of course that the reaction time for shorter gusts is constrained by sensor delays and actuator rates of the flight control system. The amount of rudder deflection depends on the yaw damper gains $k_{r}$. In the undamped case $\left(k_{r}=0.0\right)$ no rudder action is observed, leading to a significantly larger response of the heading angle. It should also be noted that the rudder deflections are well within the limits of the RTLU. Figure 8 depicts for the three different yaw damper gains the individual traces of the correlated bending and torsion moments for various gust gradient lengths at the VTP root. The envelopes are determined by a 2D convex hull of all traces including their mirrored counterpart, i.e. gust from the left and from the right hand side. The loads envelopes in Figure 8 show that more rudder deflection helps to decrease the bending moment at the cost of an increase in torsion moment. The discrete gust is usually more critical for the bending moment, so the yaw damper helps in this case. The longer gradient distances usually result in higher bending moment loads. However, the longest gust gradient distance is not necessarily the most critical one, underlining the necessity for the gust tuning to determine the sizing load cases.

\section{B. Yawing Manoeuvre}

The yawing manoeuver consist of four individual phases spanning the correlated loads envelope. Fist the initial onset of the rudder deflection causes a negative bending moment with an associated positive torsion. Depending on magnitude of the yaw damping gain, the initial rudder deflection is taken back to reduce the overshoot of the sideslip angle. The maximum sideslip angle is the second characteristic point spanning the loads envelope, where the maximum 


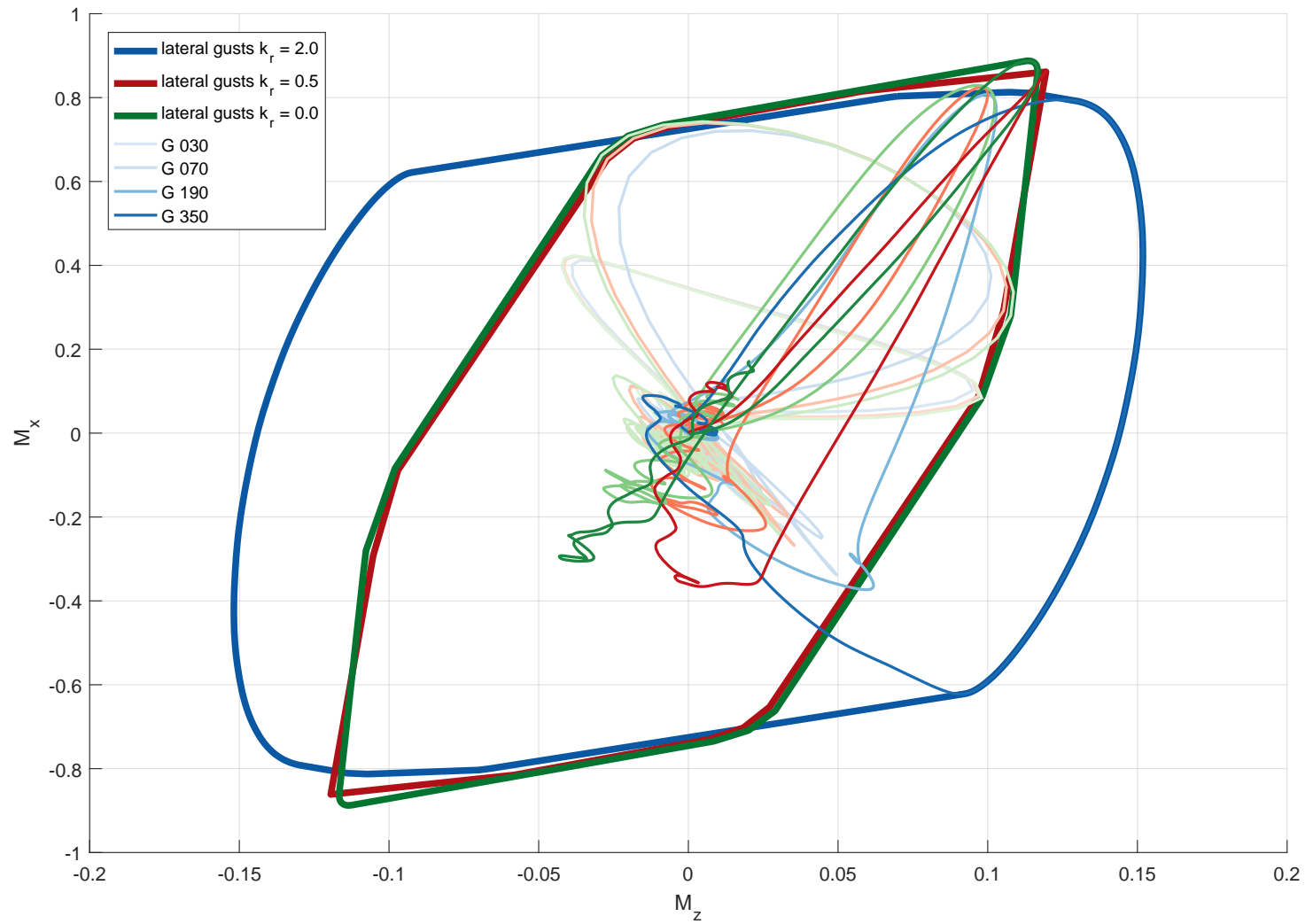

Fig. 8 Correlated bending torsion moment loads for lateral discrete gust with different yaw damping gains 


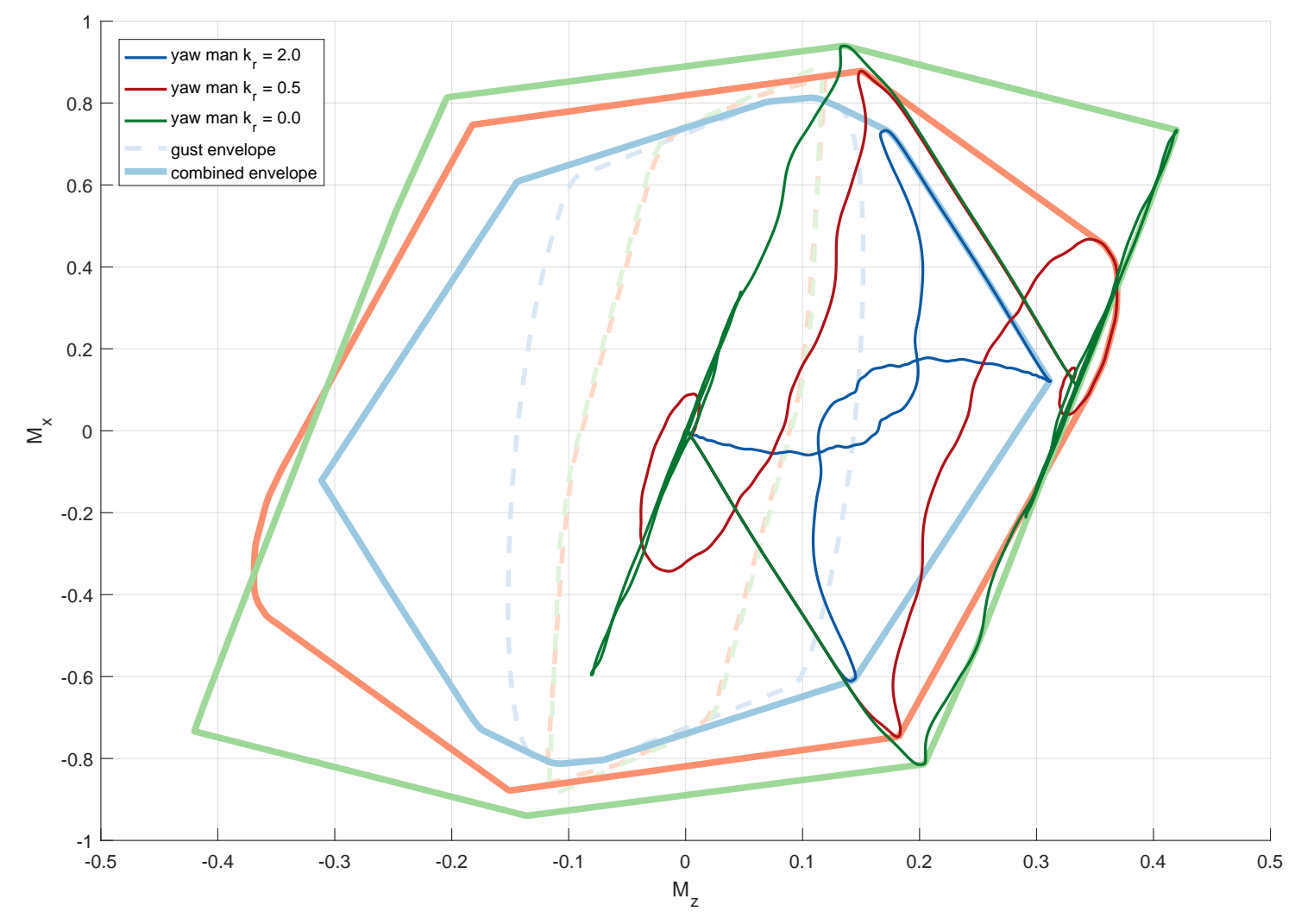

Fig. 9 Correlated bending torsion moment loads for yawing manoeuvre with different yaw damping gains

torsion moment occurs. When the oscillations of the overshoot subside, a steady sideslip angle establishes. This constitutes phase three of the yawing manoeuvre. If the yaw damper critically damps the buildup of the sideslip, this phase coincides with the previous point in the envelope. In phase four, the pedal input is returned to neutral. This is the phase where the maximum bending moment occurs, since the counteracting force due to rudder deflection is suddenly absent. This seems somewhat counterintuitive but can be regarded as an opposite rudder command superimposed with the already prevailing bending moment load due to the steady sideslip angle.

The graphs for rudder deflection $\delta_{r}$, sideslip angle $\beta$, and the bending and torsion loads $M_{x}$ and $M_{z}$ versus time can be found in Figure 10.

The loads resulting from the yawing manoeuvre are heavily influenced by the flight control system. High gains in the yaw damping function inhibit the $\beta$ overswing. The largest sideslip angle $\beta$ is responsible for the maximum torsion moment. Further, the RTLU sets limits to the allowable rudder deflection, which reduces the maximum achievable side slip angle and hence the bending and torsion loads. In phase four of the yawing manoeuvre, when the pilot command returns to neutral, the maximum bending moment is induced. This is also directly related to the maximum allowable rudder deflection, since the maximum bending moment occurs due to superposition of the achievable sideslip and the missing counter force of the rudder.

Reducing the allowable rudder deflections reliefs the loads on the vertical tail, however, this has to be balanced with the handling requirements where sufficient rudder deflection is necessary to counteract the yawing moment of a one engine inoperative condition, as well as for turn coordination. Figure 9 shows the traces of the correlated loads of the yawing manoeuvre for the different yaw damper gains. When combining the envelopes of manoeuvre and discrete gusts confirms the trends explained above - higher yaw damping gains reduce the overall loads. Also note that for the higher yaw damping $k_{r}=2.0$ the discrete gust becomes critical for the bending moment. For the intermediate damping $k_{r}=0.5$, the maximum bending moments of manoeurvre and gusts are approximately on par. For the undamped case $k_{r}=0.0$ the bending is dominated by the manoeuvre. 

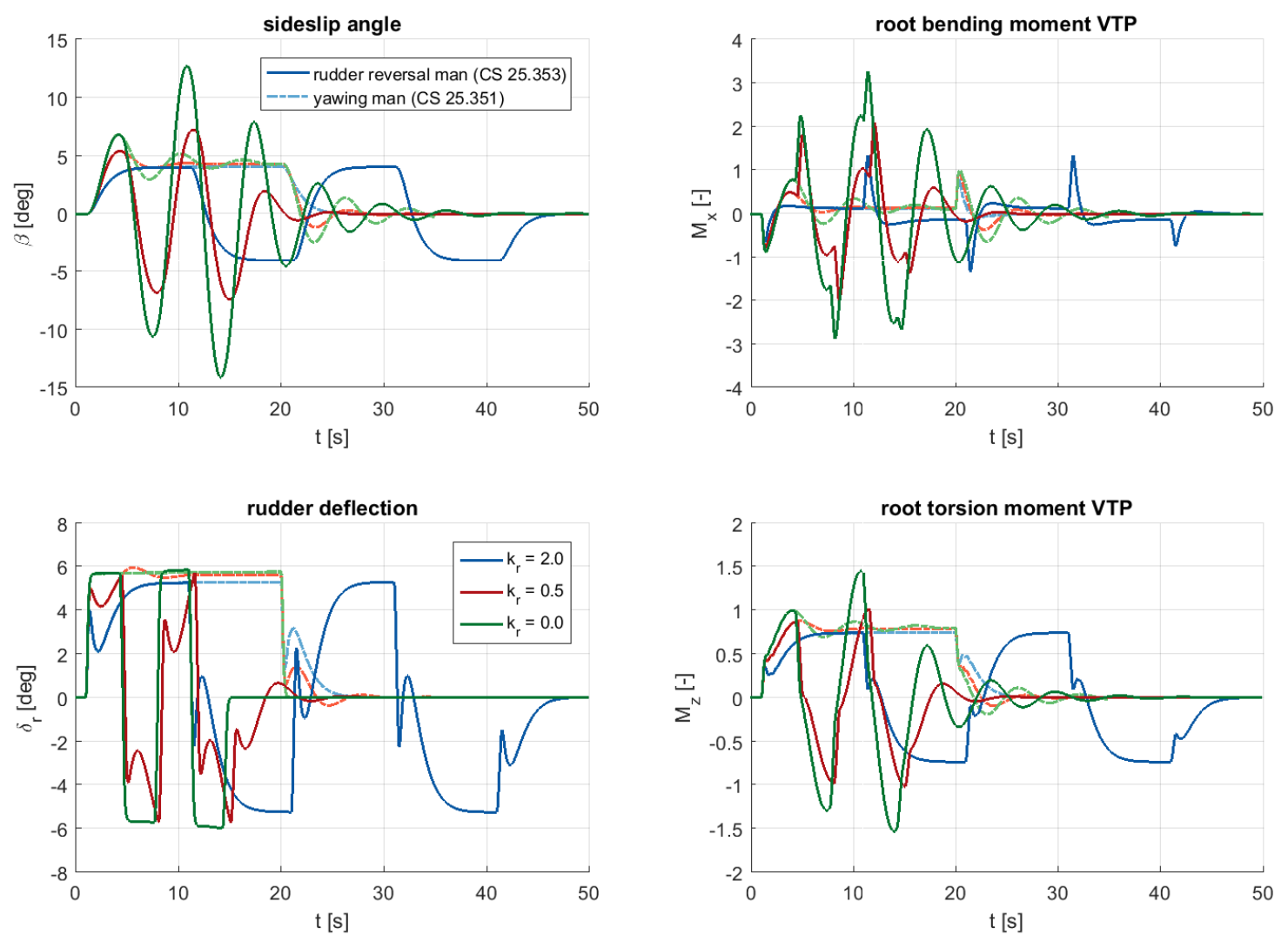

Fig. 10 Rudder Reversal Responses: sideslip rudder deflection and bending and torsion moment loads with different yaw damping gains

\section{Rudder Reversal}

The newly introduced rudder reversal condition CS 25.353 consists of two full rudder doublets in contrast to the single pedal input of the conventional yawing manoeuvre condition of CS 25.351. Figure 10 depicts the rudder deflection, the sideslip angle and the resulting bending and torsion loads for the rudder control reversal as well as the conventional yawing manoeuvre.

The initial onset of the rudder reversal condition is equal to the conventional yawing manoeuvre. Also, the rudder return causes the highest loads, just like in the conventional yaw manoeuvre, but this time the pedal input is not only commanded to return to neutral but to the maximum opposite deflection, which results in extremely large bending moments.

Furthermore, the reversing pedal input is required to be initiated at the maximum sideslip angle, instead of the steady state value. When overcritical yaw damping gains $\left(k_{r}=2.0\right)$ are employed, the successive opposite deflections do not result in any higher loads compared to a single doublet and simply follow the same trajectory in the correlated loads plot a second time. However, in the case of low yaw damping, this leads to extremely high loads. For aircraft types with a high inertia around the z-axis, these loads are so large that even when considered as ultimate loads, they are most likely prohibitive for any kind of lateral control law design with undercritical yaw damping.

Figure 11 shows the bending torsion correlated loads envelopes without the new rudder reversal condition. The traces of the rudder reversal manoeuvres are scaled down by a factor of 1.5 to constitute limit loads to assess their impact on design loads envelopes. In the case of the critically damped case, the correlated loads envelope is not expanded by a large amount and most likely covered by either other load conditions, or not completely exhausted reserve factors of the structure. For the lower yaw damping gains the loads for heavy aircraft types induced by the new load condition are severe and most likely have impact on the lateral control law design. It should be noted that this assessment is based on generic aircraft data and not on a particular aircraft or real aircraft control system and by no means conclusive. It simply is meant to show the impact of the new paragraph on the resulting loads levels and on control law design strategies. 


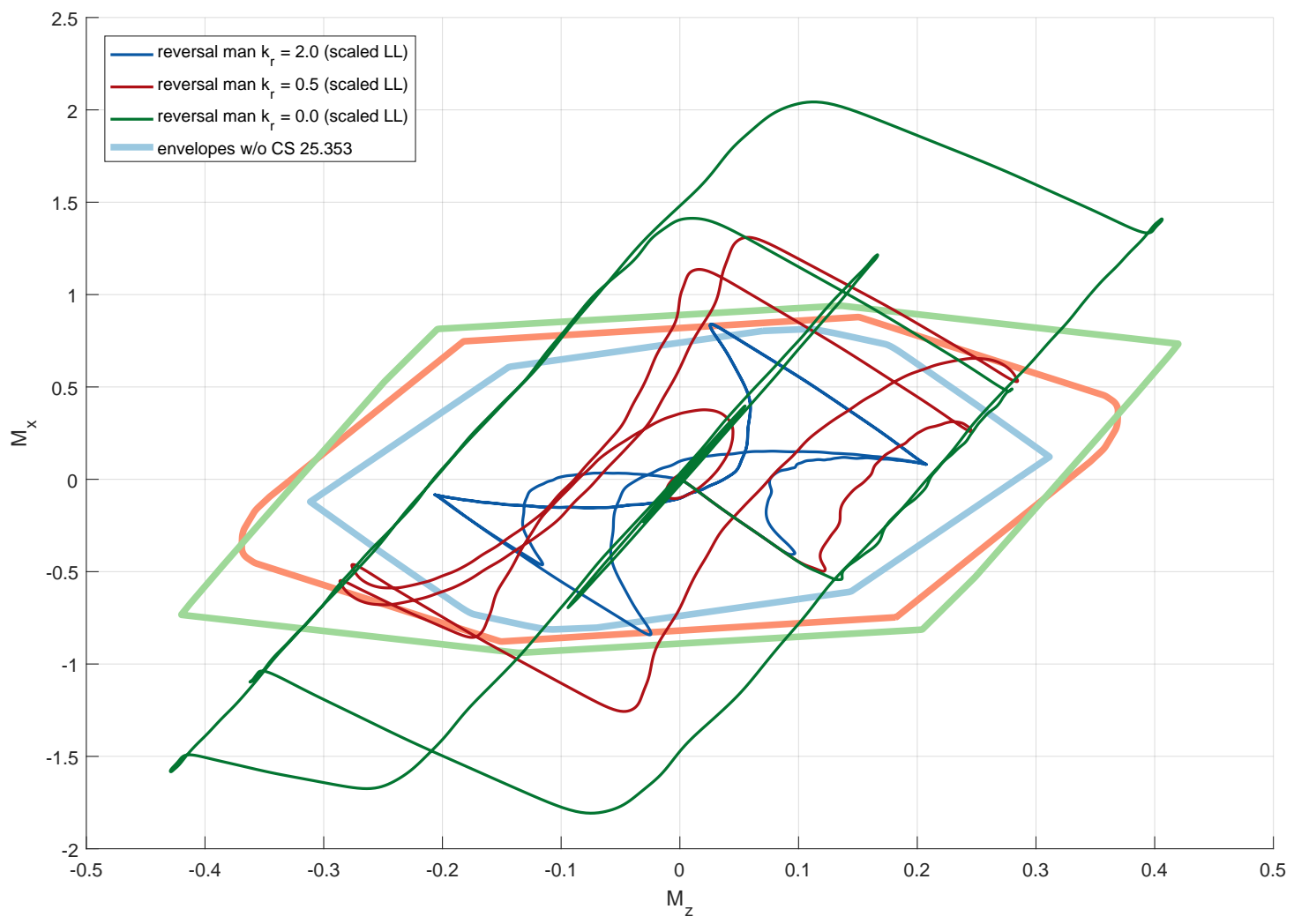

Fig. 11 Correlated bending torsion moment loads for rudder control reversal manoeuvre with different yaw damping gains 
In our paper [5] further analyses of the loads hierarchy along the VTP component axis is discussed. Furthermore, loads resulting from wake vortex encounters as compared with the new load condition are analysed.

\section{Lateral-directional control functions}

The first generation of fly-by-wire aircraft provided command augmentation and envelope protection features for the roll and pitch axes. Directional control augmentation was limited to a yaw damper and a dynamic pressure-dependent limitation of rudder travel. The latest generation of full fly-by-wire aircraft also provide command augmentation around the yaw axis by means of side slip or lateral load factor command following.

In this section, such a function is described based on the increasingly popular design methods Nonlinear Dynamic Inversion (NDI) and Incremental Nonlinear Dynamic Inversion (INDI). Since not all readers may be familiar with the control methods, we will give a simplified, single-input single-output (SISO) derivation in order to clarify the basic principles. It is noted that the control laws presented in the subsequent loads analyses are based on the full MIMO derivation and include many design-related details not presented here.

As a start, the equation of motion around the vertical axis of the aircraft may be reduced to:

$$
I_{z z} \dot{r}_{b}=q_{\infty} S c_{r e f}\left(C_{n_{\beta}} \beta+C_{n_{p}} \frac{p_{b} c_{r e f}}{V_{\mathrm{TAS}}}+C_{n_{r}} \frac{r_{b} c_{r e f}}{V_{\mathrm{TAS}}}+\cdots+C_{n_{\delta_{r}}} \delta_{r}\right)
$$

where $I_{z z}$ is the moment of inertia around the aircraft yaw axis, $r_{b}$ is the body yaw rate, $S$ the wing area, $c_{r e f}$ a reference length (in this case, mean aerodynamic choord), $C_{n} \ldots$ are stability coefficients that directly correlate with entries of the AIC matrices revisited in Secion II. In this highly simplified derivation it is assumed that only the rudder deflection $\delta_{r}$ influences the yawing moment.

In this example, the basic principle of NDI is to compute the (momentarily) required rudder deflection $\delta_{r_{c o m}}$ to achieve a commanded value $r_{b}^{\prime}$ for the yaw acceleration $\dot{r}_{b}$, so that $\dot{r}_{b} \approx r_{b}^{\prime}$. A simple way to achieve this is to just invert equation 13:

$$
\delta_{r_{c o m}}=\frac{1}{q_{\infty} S c_{r e f} C_{n_{\delta_{r}}}}\left[I_{z z} r_{b}^{\prime}-q_{\infty} S c_{r e f}\left(C_{n_{\beta}} \beta+C_{n_{p}} \frac{p_{b} c_{r e f}}{V_{\mathrm{TAS}}}+C_{n_{r}} \frac{r_{b} c_{r e f}}{V_{\mathrm{TAS}}}+\ldots\right)\right]
$$

Inserting this equation into (13) yields $\dot{r}_{b}=r_{b}^{\prime}$. Since $(14)$ is a control law and $(13)$ represents the actual flight dynamics, this equation will only hold approximately in practice. It is important to note that the variables $\beta, p_{b}, q_{\infty}$, etc. are obtained from measurement.

Starting from this principle, it is possible to wrap further control laws around this core. For example:

$$
r_{b}^{\prime}=K_{r}(s)\left(r_{b_{c o m}}-r_{b}\right)
$$

where the commanded yaw rate $r_{b_{c o m}}$ in turn is computed from an inversion-based control law for the side slip angle $\beta$ (not detailed here). The linear controller transfer function usually is a PID law, but it may be of any type. This loop is nearly always linear, as nonlinearities are compensated by the model inversion: this is why NDI is a highly popular method to handle nonlinearities in flight control.

In order to complete the derivation, the commanded yaw rate is computed from inversion of the lateral force equation w.r.t. $r_{b}$ and using $\dot{\beta}$ as a new command variable $\beta^{\prime}$ :

$$
\dot{\beta} \approx p_{b} \sin \alpha-r_{b} \cos \alpha+\frac{g}{V_{\mathrm{TAS}}} \sin \phi+\frac{q_{\infty} S}{m V_{\mathrm{TAS}}}\left(C_{Y_{0}}+C_{Y_{\beta}} \beta+\ldots\right)
$$

Side slip control law is completed by:

$$
\beta^{\prime}=K_{\beta}(s)\left(\beta_{\text {com }}-\beta_{\text {filt }}\right)
$$

where $\beta_{\text {filt }}$ equals complementarily filtered airdata and inertial measurements. As already said, the above equations are provided for completeness, but will not be further discussed in the following.

There is another way to look at the NDI control law in eqn. (14). The term

$$
q_{\infty} S c_{r e f}\left(C_{n_{\beta}} \beta+C_{n_{p}} \frac{p_{b} c_{r e f}}{V_{\mathrm{TAS}}}+C_{n_{r}} \frac{r_{b} c_{r e f}}{V_{\mathrm{TAS}}}+\ldots\right)=f\left(q_{\infty}, \beta, \ldots\right)
$$

is basically the total yawing moment due to aerodynamic flow, without the influence of the rudder. From this perspective, one might think of measuring this moment by means of an angular accelerometer, subtracting the rudder influence by 
means of a computed estimate. This approach was already presented in a paper by Smith in 1998 [31]. Using eqn. [13], this results in:

$$
f\left(q_{\infty}, \beta, \ldots\right)=I_{z z} \dot{r}_{b}-q_{\infty} S c_{r e f} C_{n_{\delta_{r}}} \delta_{r}
$$

where now $\dot{r}_{b}, q_{\infty}$ and $\delta_{r}$ are replaced by measured or estimated values $\dot{r}_{b_{\text {meas }}}, q_{\infty_{\text {meas }}}$ and $\delta_{r_{\text {meas }}}$ respectively. Substituting this equation into eqn. (14) gives:

$$
\delta_{r_{c o m}}=\frac{I_{z z}}{q_{\infty} S c_{r e f} C_{n_{\delta_{r}}}}\left(r_{b}^{\prime}-\dot{r}_{\text {meas }}\right)+\delta_{r_{\text {meas }}}
$$

This very simple control law basically computes increments on the current actual control deflection and only requires angular acceleration, dynamic pressure and the current control deflection as measurements. The great advantage of this incremental nonlinear dynamic inversion (INDI [32]) approach is its simplicity and very low dependence on model data for achieving excellent tracking performance, as compared with NDI.

It is crucial that $\delta_{r_{\text {meas }}}$ and $\dot{r}_{\text {meas }}$ are synchronous in terms of measurement delay and filtering, as otherwise eqn. (19) is simply inaccurate. This synchronization principle was found at DLR during the first practical implementation of INDI on a flight vehcile (FASER UAV, operated by the University of Minnesota) and is actually what has made INDI work robustly in practice. In 2017, DLR and TU-Delft performed the very first flight test on a CS-25 class aircraft, demonstrating the validity of this control approach. During this first and also later implementations, it was found that estimation of the the angular acceleration and control deflections works nearly just as well, with little loss of performance.

One aspect that has received little to no attention in literature on INDI so far, is control activity and structural loading. One of the key trade-offs in flight control law design is between accurate tracking performance and control activity. The latter always goes at the cost of energy consumption and wear in hydraulic or electric actuators. This becomes even more important if engine throttle controls are involved. Control activity is immediately influenced by stability margins and control bandwith. In case of flight controls, sensors are also directly influenced by structural dynamics at the attachment points and even more explicit, by aerodynamic disturbances sensed by the airdata sensors. For inertial sensors, various types of filters (low pass, notch, etc.) are used to avoid structural dynamics to propagate unintentionally into the (primary) control laws. For airdata, complementary filtering is used: airdata and inertial measurements are filtered complementarily in order to remove higher frequency disturbances without losing phase margin in the feedback loops.

When looking at the control law in 20 it immediately becomes clear that there is little possibility to filter out aerodynamic disturbances as compared with (14). By using angular acceleration measurements, the control system senses and will try to compensate for basically all external disturbances. In case of NDI, these disturbances enter the equation via the side slip (and any other air data) measurements and complementary filtering allows for directly trading between disturbance rejection and control activity [33]. For this reason, it is very interesting to have a closer look at loads induced by INDI control laws, especially in comparison with NDI. Furthermore, it will be clear that angular acceleration measurement will be far more susceptible to structural dynamics, requiring careful filter design to avoid unintended loads and deterioration of flutter stability.

\section{Comparison of control algorithms}

In this section, four types of control algorithms will be compared with the open loop case:

- Yaw damper with gain $k_{r}=0.0$, see Section II.D.1('open loop');

- Yaw damper with gain $k_{r}=2.0$;

- NDI-based control law (side slip command);

- INDI-based control law (side slip command);

It is further noted that the rudder travel limitation (RTL) applies to all algorithms. Also, the NDI and INDI control functions are part of a fully integrated multi-input multi-output control system that provides command augmantation around the pitch and roll axes as well.

First of all, it is interesting to compare the yawing and rudder control reversal manoeuvres. The side slip angle, rudder deflection, root bending and torsion moment responses are shown in Figures 12. All control laws (apart from open loop) provide smooth side slip command responses. Those resulting from the NDI and INDI control laws have a considerable delay, which is caused by the inner ( $r_{b}$ command) / outer ( $\beta$ command) loop structure described in the previous section. This structure assumes a so-called time scale separation between angular rate and aerodynamic 
attitude angle responses and imposes a limit on the bandwith in order to fulfill this assumption. This may be improved considerably, see for example [7], but this has not been done here. The faster response for the yaw damper control law is caused by the direct feed through between the pilot input and rudder deflection, see the lower left subplot. It is also interesting to note that the responses for NDI and INDI are nearly identical, which, in absence of disturbances, could have been expected.
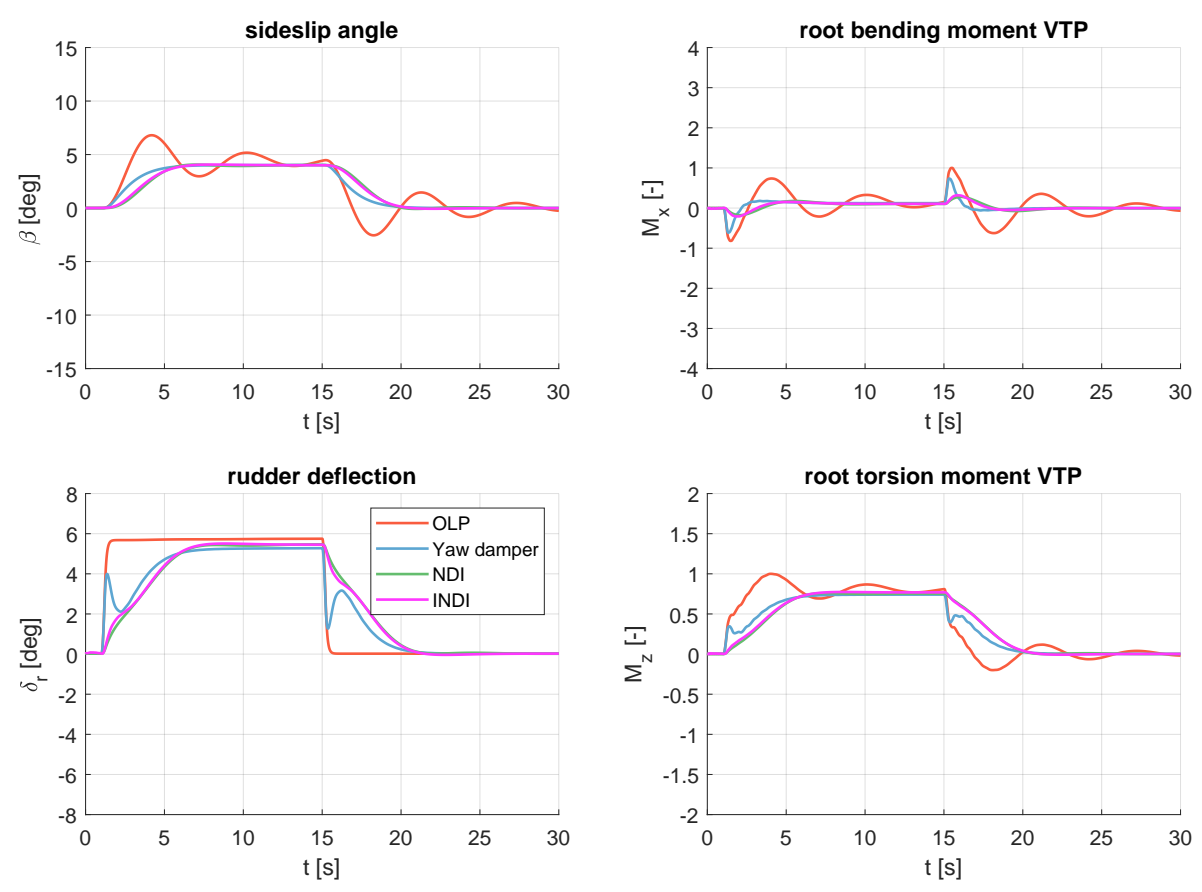

Fig. 12 Yawing manoeuvre responses for different controller types

The steady state side slip angles of NDI and INDI are based flight-condition dependent command limitation (here: $+/-4 \mathrm{deg}$ ). At this angle, the steady state rudder deflection stays just below its travel limit.

Finally, it is interesting to see that maximum torsion and bending moments at the VTP root are reduced as compared with the open loop case due to the damped and command-limited responses. The yaw damper function causes somewhat higher initial root bending moments due to aforementioned direct feed through between the pilot command and rudder deflection.

Looking at the correlated bending torsion moment loads in Figure 13 , where all closed loop envelopes are well within the open loop one.

The very same analysis can be performed for the rudder reversal case, see Figure 14. The corresponding correlated loads plots and envelopes are given in Figure 15

A very different picture arises when looking at the discrete gust responses, see Figure 16 The INDI and, to a lesser extent, the NDI control laws cause a massive initial rudder deflection that is aimed at compensating for the angular acceleration that is caused by the gust impact. As already stated in the previous section, this effect has been expected. However, in the case of NDI the measured side slip angle, which briefly carries the lateral gust impact, is filtered in combination with its inertial complement. This design degree of freedom allows for addressing the rudder response with negligible loss of trackig performance. In case of INDI, the picture is quite different. The rudder deflection resulting from the control law in eqn. 20 directly responds to the angular acceleration. Looking at the correlated bending and torsion loads in Figs. 18, 17 and 19, it becomes clear that the excellent tracking performance of INDI comes at a price. The high rudder deflection commands cause massive torsional loads at the VTP root. This issue MUST be addressed 


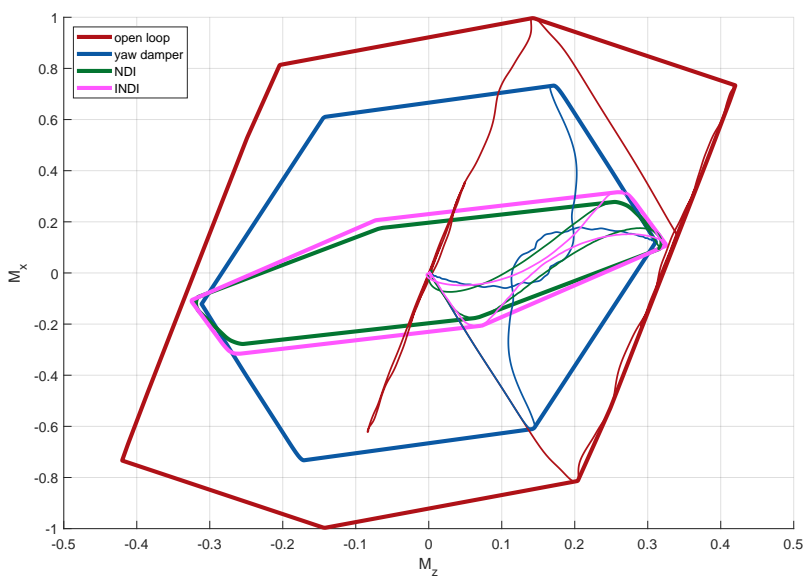

Fig. 13 Correlated bending torsion moment loads for the yawing manoeuvre: simulation responses (thin) and envelopes (fat)
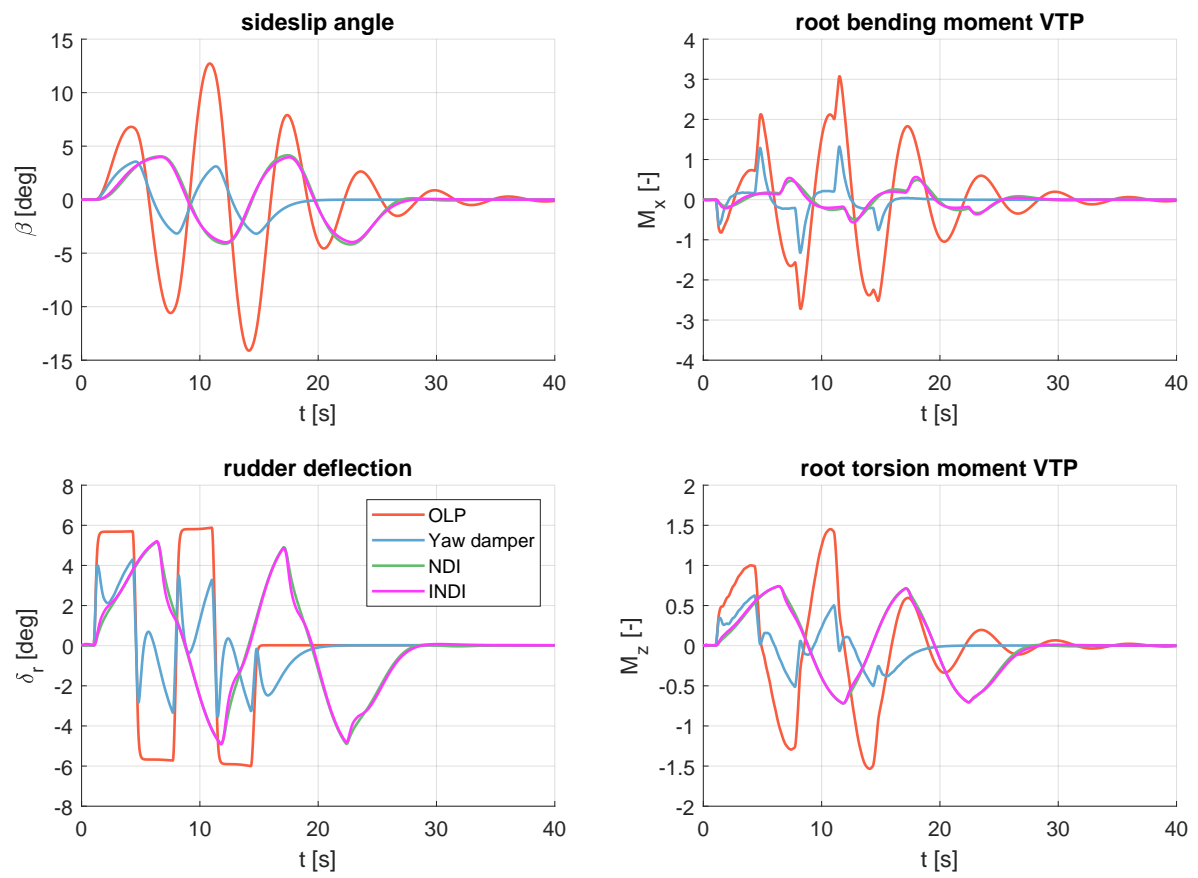

Fig. 14 Rudder control reversal manoeuvre responses for different controller types

in a flight control system design. Although not depicted in this paper, setting all gains in the outer loops to zero only marginally reduces the initial rudder deflection, so that the main focus should be on appropriate filtering / estimation of 


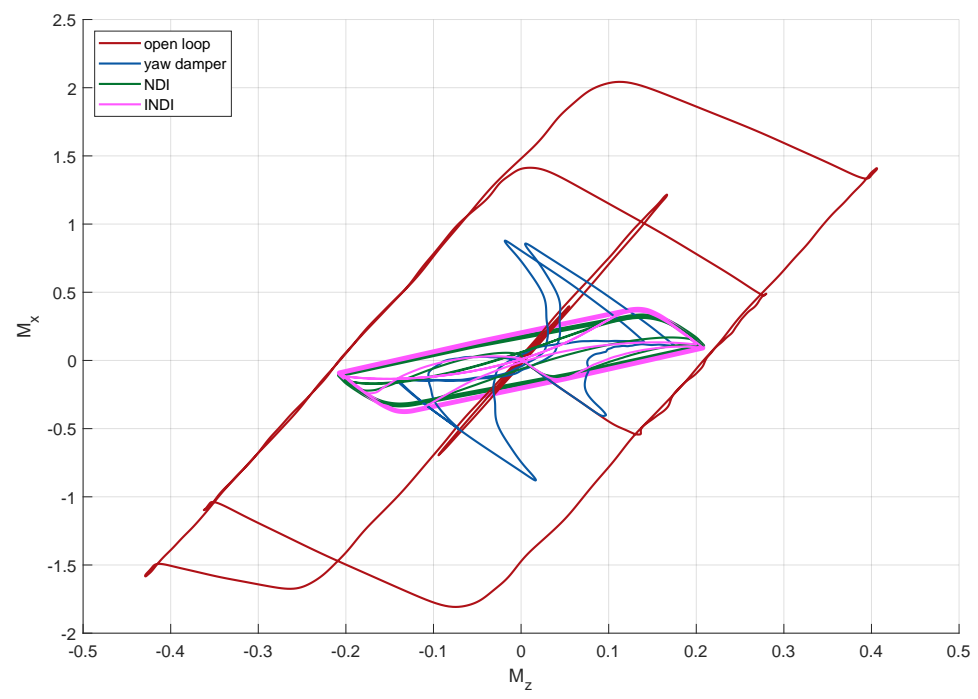

Fig. 15 Correlated bending torsion moment loads for the rudder reversal manoeuvre: simulation responses (thin) and envelopes (fat)

the angular acceleration measurements. In this example, a second order Butterworth filter with a cut-off frequency of 10 $\mathrm{rad} / \mathrm{s}$ has been applied.

Figure 20 finally shows the envelopes incorporating gust, yawing manoeuvre and rudder reversal load conditions for all control laws. Again, the INDI control law massively exceeds the envelopes of other algorithms, however, it stays (just) within the bounds of the open-loop one. 

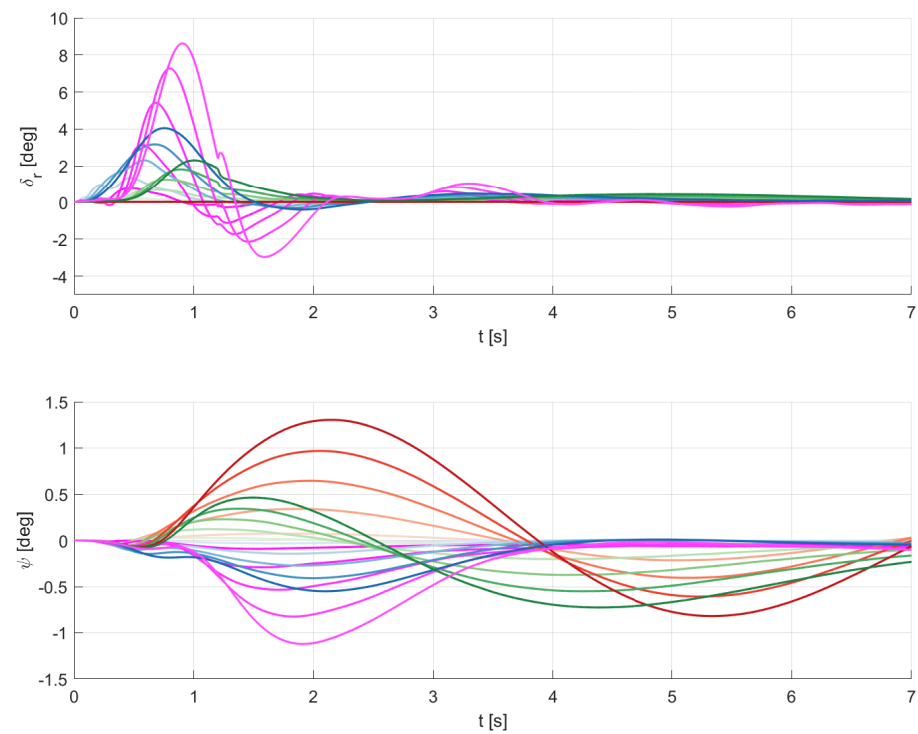

Fig. 16 Gust responses for rudder and yaw angles for different control algorithms (legend: see Fig. 15)

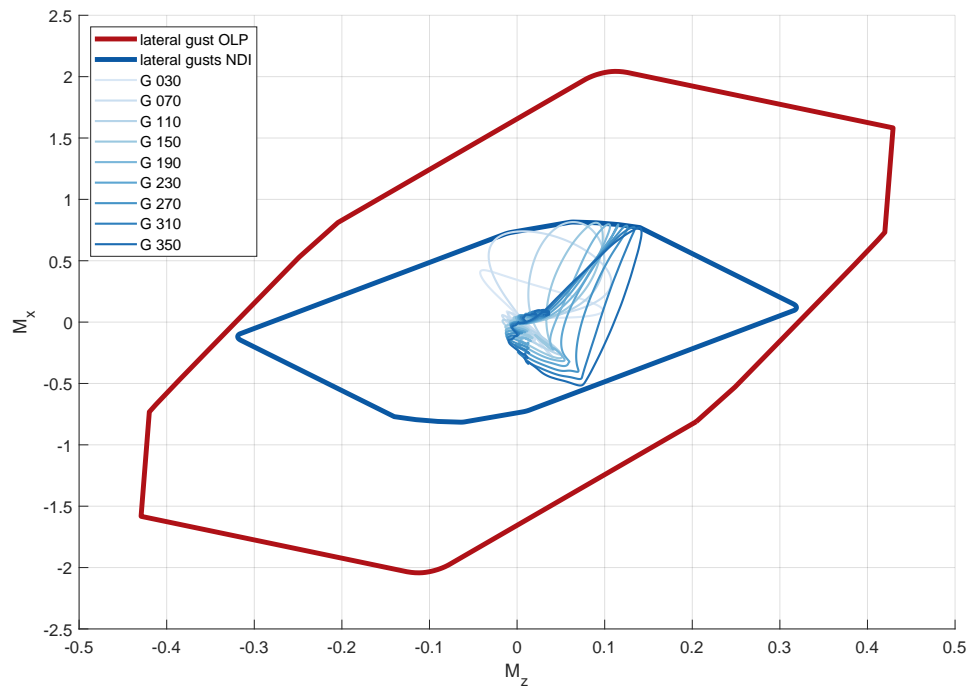

Fig. 17 Correlated bending torsion moment loads for various gust lengths for NDI 


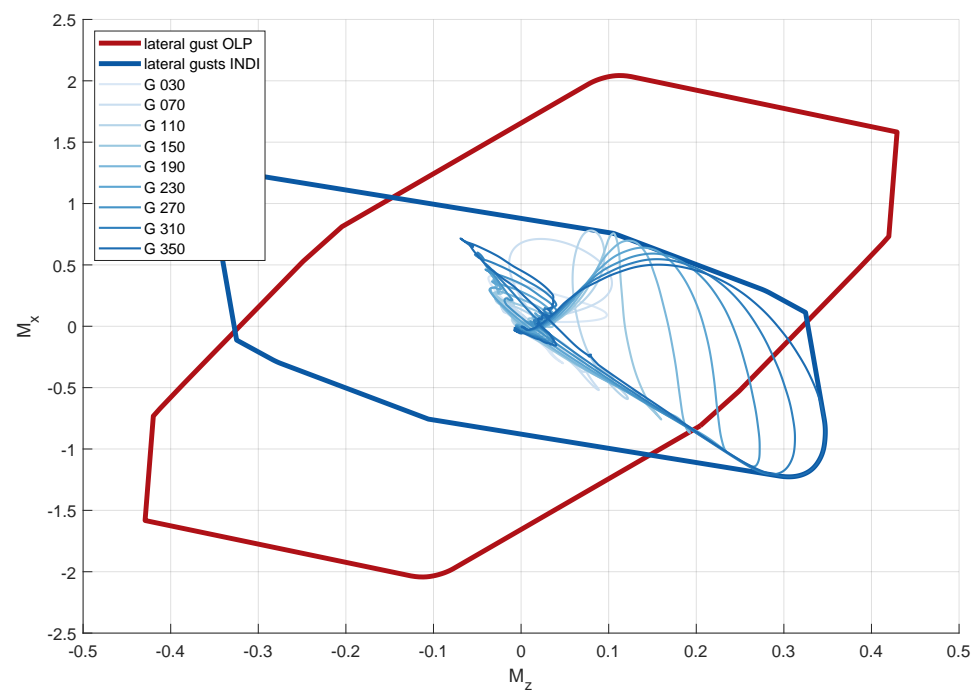

Fig. 18 Correlated bending torsion moment loads for various gust lengths for INDI

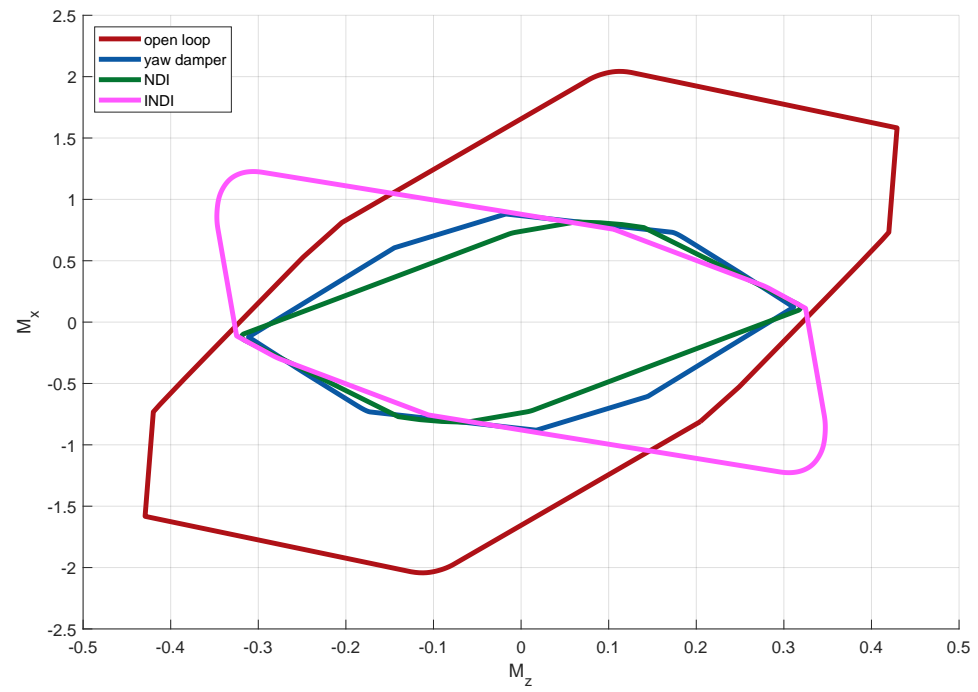

Fig. 19 Correlated bending torsion moment loads for various gust lengths for all control laws 


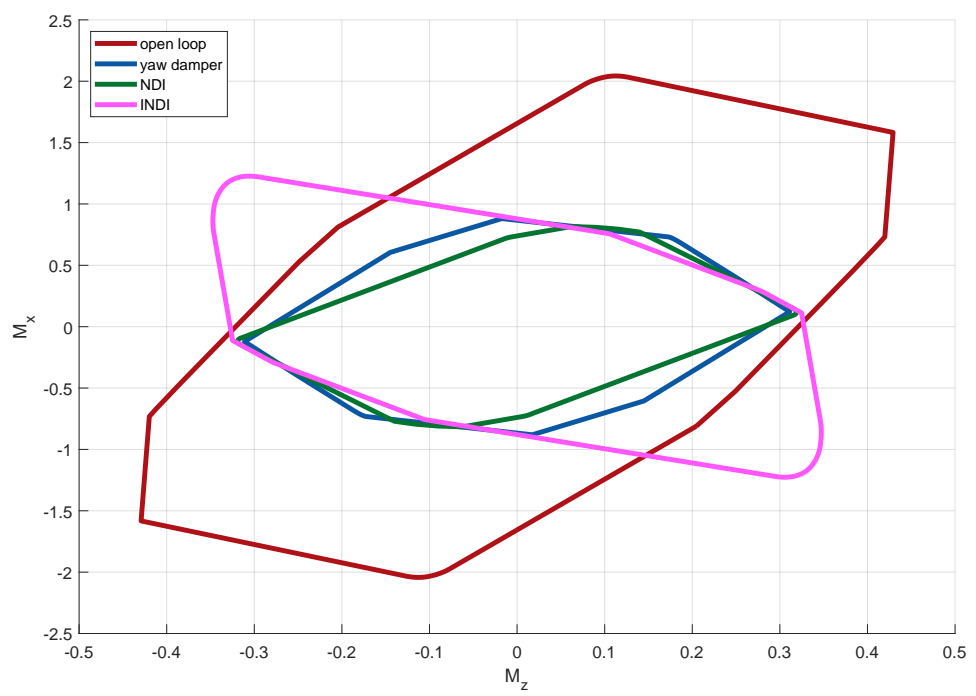

Fig. 20 Correlated bending torsion moment load envelopes for all loads conditions for all control laws 


\section{Summary and Conclusion}

An integrated model scheme to simulate manoeuvres and gusts of controlled flexible aircraft to determine design loads was presented. Complex scenarios with position and attitude dependent gust field such as wake vortex encounters are possible with this scheme. The unsteady aerodynamics can be accounted for by means of a physical rational function approximation. The present approach allows for a clear separation of quasisteady (important for manoeuvres) and unsteady aerodynamics (important for gusts and turbulence). The model equations are implemented for time domain simulations. This allows to easily account for nonlinearities in the aerodynamics or in the flight control laws, where rate and deflection limits are common.

A set of critical load conditions for the structural sizing of the vertical tail plane has been detailed: The yawing manoeuvre (CS 25.351) and the discrete lateral gust (CS 25.341(a)). Due to past flight incidents, where multiple reversing rudder pedal inputs made by pilots, a new load condition was introduced. The rudder control reversal condition (CS 25.353). The impact on the design loads of this new rule was assessed.

As all these load conditions are heavily influenced by the flight control laws, in particular by the yaw damper function and the rudder travel limitation unit. The influence of various gains with a basic yaw damper function were compared first. Next, more advanced control laws based on Nonlinear Dynamic Inversion (NDI) and it incremental derivative (INDI) were analysed. These control laws provide command augmentation around the yaw axis by means of side slip angle command following. Here a major point of attention surfaced for the INDI-based system. The inherent angular acceleration command inner-most control loop leaves little design degree of freedom for trading disturbance rejection against control activity and, in this case, considerably higher bending and torsional moment loads on the vertical tail plane. Although the computations were performed for only one generic aircraft type, it is strongly recommended to pay attention to this worrying aspect in a full system design and before flight testing on fixed-wing aircraft.

\section{References}

[1] European Aviation Safety Agency, Certification Specifications for Large Aeroplanes CS-25, Vol. Amendment 22, EASA, 2018.

[2] Transportation Safety Board of Canada, "Encounter with Wake Turbulence, Air Canada Airbus A319-114 C-GBHZ," Tech. Rep. Aviation Investigation Report A08W0007, TSB, 2008.

[3] European Aviation Safety Agency, "Unintended or inappropriate rudder usage rudder reversals," Notice of Proposed Amendment 2017-18 RMT.0397, EASA, 2017.

[4] Federal Aviation Administration, "Yaw Maneuver Conditions Rudder Reversals," Notice of proposed rulemaking 18-04 FAA20180653, FAA, 2018.

[5] Kier, T., Müller, R., and Looye, G., "An Integrated Analysis Model for Assessment of Critical Load Conditions for the Vertical Tail Plane,” International Forum on Aeroelasticity and Structural Dynamics, IFASD 2019, 2019.

[6] Grondman, F., Looye, G., Kuchar, R., Chu, Q. P., and van Kampen, E.-J., "Design and Flight Testing of Incremental Backstepping based Control Laws with Angular Accelerometer Feedback," AIAA SciTech Forum, 2018.

[7] Keijzer, T., Looye, G., Chu, Q. P., and van Kampen, E.-J., "Design and Flight Testing of Incremental Backstepping based Control Laws with Angular Accelerometer Feedback," AIAA SciTech Forum, 2019. doi: https://doi.org/10.2514/6.2019-0129.

[8] Wang, X., van Kampen, E.-J., Chu, Q., and De Breuker, R., "Flexible Aircraft Gust Load Alleviation with Incremental Nonlinear Dynamic Inversion," Journal of Guidance, Control, and Dynamics: devoted to the technology of dynamics and control, Vol. 42, No. 7, 2019, pp. 1519-1536. doi: https://doi.org/10.2514/1.G003980.

[9] Kier, T., and Looye, G., "Unifying Manoeuvre and Gust Loads Analysis," International Forum on Aeroelasticity and Structural Dynamics, 2009.

[10] Kier, T., "An Integrated Loads Analysis Model including Unsteady Aerodynamic Effects for Position and Attitude dependent Gust Fields," International Forum on Aeroelasticity and Structural Dynamics, 2011.

[11] Hofstee, J., Kier, T., Cerulli, C., and Looye, G., “A Variable, Fully Flexible Dynamic Response Tool for Special Investigations (VarLoads)," International Forum on Aeroelasticity and Structural Dynamics, 2003.

[12] Guyan, R. J., "Reduction of stiffness and mass matrices," Journal of Aircraft, Vol. 3, No. 2, 1965, p. 380. doi: https://doi.org/ $10.2514 / 3.2874$ 
[13] Waszak, M. R., and Schmidt, D. K., "On the Flight Dynamics of Aeroelastic Vehicles," AIAA Atmospheric Flight Mechanics Conference, AIAA, 1986, pp. 120-133. doi: https://doi.org/10.2514/6.1986-2077.

[14] M. R. Waszak and D. K. Schmidt, "Flight Dynamics of Aeroelastic Vehicles," Journal of Aircraft, Vol. 25, No. 6, 1988, pp. 563-571. doi: https://doi.org/10.2514/3.45623.

[15] R. L. Bisplinghoff, H. Ashley, R. L. Halfman, Aeroelasticity, Dover Publications Inc., 1955.

[16] Roger, K. L., "Airplane Math Modeling Methods for Active Control Design," AGARD Structures and Materials Panel, AGARD, 1977, pp. 4-1 - 4-11.

[17] Pistolesi, E., "Betrachtungen "uber die gegenseitige Beeinflussung von Tragfl"ugelsystemen," Gesammelte Vortr"age der Hauptversammlung 1937 der Lilienthal Gesellschaft, 1937.

[18] Harder, R., and Desmarais, R., "Interpolation Using Surface Splines,” Journal of Aircraft, Vol. 9, No. 2, 1972 , pp. $189-191$. doi: https://doi.org/10.2514/3.44330.

[19] Rodden, W., Taylor, P., and Jr., S. M., "Further Refinement of the Subsonic Doublet-Lattice Method," Journal of Aircraft, Vol. 9, No. 10, 1998, pp. 693-702. doi: https://doi.org/10.2514/2.2382.

[20] Edwards, J. W., "Applications of Laplace transform methods to airfoil motion and stability calculations," 20th Structures, Structural Dynamics and Materials Conference, 1979. doi: https://doi.org/10.2514/6.1979-772.

[21] Abel, I., "An analytical technique for predicting the characteristics of a flexible wing equipped with an active flutter-suppression system and comparison with wind-tunnel data," Tech. Rep. NASA TP-1367, NASA LARC, 1979.

[22] Collar, A., “The Expanding Domain of Aeroelasticity," Journal of the Royal Aeronautical Society, Vol. 50, 1946, pp. 613-636.

[23] Lambregts, A., Total energy based flight control system, US Patent 4536843, 1985.

[24] Lambregts, A. A., “TECS Generalized Airplane Control System Design-An Update,” Advances in Aerospace Guidance, Navigation and Control, Springer, 2013, pp. 503-534.

[25] Looye, G., "TECS/THCS-based generic autopilot control laws for aircraft mission simulation," Advances in Aerospace Guidance, Navigation and Control, 2013.

[26] Pratt, K., and Walker, W., "A revised gust-load formula and a re-evaluation of v-g data taken on civil transport airplanes from 1933 to 1950,” Tech. Rep. NACA Report 1206, NACA, 1954.

[27] Joint Aviation Authorities, Joint Aviation Requirements JAR-25 Large Aeroplanes, Vol. Change 13, JAA, 1989.

[28] Joint Aviation Authorities, Joint Aviation Requirements JAR-25 Large Aeroplanes, Vol. Change 14, JAA, 1994.

[29] Barnes, T. J., "Harmonization of US and European Gust Criteria for Transport Airplanes," 17th International Congress of the Aeronautical Sciences, Stockholm, Sweden, September 9-14. 1990, ICAS, 1990.

[30] European Aviation Safety Agency, Certification Specifications for Large Aeroplanes CS-25, Vol. Amendment 21, EASA, 2018.

[31] Smith, P., A simplified approach to nonlinear dynamic inversion based flight control, ???? doi: https://doi.org/10.2514/6.19984461.

[32] Sieberling, S., Chu, Q. P., and Mulder, J. A., "Robust Flight Control Using Incremental Nonlinear Dynamic Inversion and Angular Acceleration Prediction," Journal of Guidance, Control, and Dynamics, Vol. 33, No. 6, 2010, pp. 1732-1742. doi: https://doi.org/10.2514/1.49978.

[33] "An integrated Approach to Aircraft Modelling and Flight Control Law Design, Author = Looye, G.H.N., School = Delft University of Technology, Year = 2008," Ph.D. thesis. 